\title{
Paraventricular Thalamic Nucleus: Subcortical Connections and Innervation by Serotonin, Orexin, and Corticotropin-Releasing Hormone in Macaque Monkeys
}

\author{
DAVID T. HSU ${ }^{1}$ AND JOSEPH L. PRICE ${ }^{2 *}$ \\ ${ }^{1}$ Molecular and Behavioral Neuroscience Institute, University of Michigan, Ann Arbor, Michigan 48109 \\ ${ }^{2}$ Department of Anatomy and Neurobiology, Washington University School of Medicine, St. Louis, Missouri 63110
}

\begin{abstract}
The present study examines subcortical connections of paraventricular thalamic nucleus $(\mathrm{Pa})$ following small anterograde and retrograde tracer injections in cynomolgus monkeys (Macaca fascicularis). An anterograde tracer injection into the dorsal midline thalamus revealed strong projections to the accumbens nucleus, basal amygdala, lateral septum, and hypothalamus. Retrograde tracer injections into these areas labeled neurons specifically in Pa. Following a retrograde tracer injection into $\mathrm{Pa}$, labeled neurons were found in the hypothalamus, dorsal raphe, and periaqueductal gray. Pa contained a remarkably high density of axons and axonal varicosities immunoreactive for serotonin $(5-\mathrm{HT})$ and orexin/hypocretin (ORX), as well as a moderate
\end{abstract}

density of fibers immunoreactive for corticotropin-releasing hormone (CRH). A retrograde tracer injection into $\mathrm{Pa}$ combined with immunohistochemistry demonstrated that ORX and 5-HT axons originate from neurons in the hypothalamus and midbrain. Pa-projecting neurons were localized in the same nuclei of the hypothalamus, amygdala, and midbrain as CRH neurons, although no double labeling was found. The connections of $\mathrm{Pa}$ and its innervation by 5-HT, ORX, and CRH suggest that it may relay stress signals between the midbrain and hypothalamus with the accumbens nucleus, basal amygdala, and subgenual cortex as part of a circuit that manages stress and possibly stress-related psychopathologies. J. Comp. Neurol. 512:825-848, 2009.

๑) 2008 Wiley-Liss, Inc.

Indexing terms: stress; depression; mood disorders; amygdala; accumbens nucleus; caudate nucleus; hypothalamus; periaqueductal gray; paratenial nucleus; striatum

The paraventricular thalamic nucleus $(\mathrm{Pa})$ is part of the midline and intralaminar group of thalamic nuclei, although it differs from the other midline nuclei because of its developmental origin as part of the epithalamus, and it has a specific chemical signature (Jones, 2007). The midline nuclei have previously been thought to project diffusely and nonspecifically to the cerebral cortex (Jones and Leavitt, 1974; Royce et al., 1989), and it has even been questioned whether $\mathrm{Pa}$ has a cortical projection at all (Jones, 2007). With the advent of more refined tracing techniques, however, it is clear that $\mathrm{Pa}$ and other midline and intralaminar nuclei have specific projections to restricted regions of the cerebral cortex (Berendse and Groenewegen, 1991; Hsu and Price, 2007), and the striatum (Beckstead, 1984; Berendse and Groenewegen, 1990).

$A$ recent study in primates found that $\mathrm{Pa}$ is strongly and specifically connected with the cortex ventral to the genu of the corpus callosum, especially area 25 (Hsu and Price, 2007). The "subgenual" cortex exhibits abnormal activity in major depressive disorder, as shown by functional neuroimaging (e.g., Drevets et al., 1997; Mayberg et al., 1999; Pizzagalli et al., 2004; Périco et al., 2005; Siegle et al., 2006; Van Laere et al., 2006), with reversal of these changes after successful treatment (Mayberg et al., 1999; Brody et al., 2001). In one remarkable study, white matter near the subgenual cortex was targeted for deep brain stimulation for severe, intractable depression, resulting in sustained symptom remission in the majority of patients (Mayberg et al., 2005). Another recent study showed that during rest the coupling of neuronal oscillatory activity between the midline thalamus and subgenual area 25 was greater in depressed subjects compared to controls, suggesting an abnormal functional link between the two (Greicius et al., 2007).

$\mathrm{Pa}$ is particularly sensitive to stressors. In rats, levels of the molecular marker c-fos are consistently and strongly increased specifically in $\mathrm{Pa}$ following several types of physical and psychological stressors (e.g., Chastrette et al., 1991;

Grant sponsor: National Institutes of Health; Grant numbers: MH070941, MH068148.

*Correspondence to: Joseph L. Price, Department of Anatomy \& Neurobiology, Campus Box 8108, Washington University School of Medicine, 660 S. Euclid Ave., St. Louis, MO 63110. E-mail: pricej@pcg.wustl.edu

Received 19 November 2007; Revised 29 August 2008; Accepted 2 November 2008

DOI 10.1002/cne.21934

Published online in Wiley InterScience (www.interscience.wiley.com). 
Sharp et al., 1991; Imaki et al., 1993; Cullinan et al., 1995; Spencer et al., 2004). Furthermore, lesion studies indicate that $\mathrm{Pa}$ is uniquely involved in the neuroendocrine and behavioral adaptation to chronic, but not acute stress (Bhatnagar and Dallman, 1998; Bhatnagar et al., 2002, 2003). There is substantial evidence that increased sensitivity to stress is an important risk factor for major depression (Hasler et al., 2004), and these animal studies suggest that $\mathrm{Pa}$ may contribute to maladaptive responses to stress in the etiology and/or maintenance of mood disorders.

In the present study, subcortical connections of the primate $\mathrm{Pa}$ were examined. Serotonin (5-HT) and corticotropinreleasing hormone $(\mathrm{CRH})$, two neurotransmitter systems involved in stress responses and major depression (Nemeroff et al., 1988; Arango et al., 2002; Maier and Watkins, 2005) were examined for their inputs to $\mathrm{Pa}$. In addition, inputs to $\mathrm{Pa}$ by orexin/hypocretin (ORX) were also investigated. ORX has a dense and restricted innervation of $\mathrm{Pa}$ in rodents (Kirouac et al., 2005), and has been substantially implicated in the regulation of sleep and appetite (Sakurai, 2007).

\section{MATERIALS AND METHODS Animals}

Five Macaca fascicularis monkeys were prepared with axonal tracer injections for this study (cases OM64, OM67, OM73, OM74, and OM78). In addition, material from 13 other monkeys that were prepared for previous studies (Amaral and Price, 1984; Carmichael and Price, 1995; Öngür et al., 1998; Ferry et al., 2000) was also analyzed in relation to subcortical connections with $\mathrm{Pa}$. Because several tracers were injected in each animal, a total of 27 tracer experiments were analyzed. Animal facilities were AAALAC-approved, and all procedures were in accordance with the guidelines of the National Institutes of Health and the Institutional Animal Care and Use Committee of Washington University (St. Louis, MO).

ABBREVIATIONS

\begin{tabular}{|c|c|c|c|}
\hline 25 & Brodmann area 25 & ic & Internal capsule \\
\hline 3V & Third ventricle & IGP & Internal globus pallidus \\
\hline $4 n$ & Trochlear nerve & $\mathrm{L}$ & Lateral nucleus of the amygdala \\
\hline $5-\mathrm{HT}$ & Serotonin & LC & Locus ceruleus \\
\hline AAA & Anterior amygdaloid area & LH & Lateral hypothalamus \\
\hline ABmg & Accessory basal nucleus, magnocellular division & IPAG & Lateral periaqueductal gray \\
\hline ABpc & Accessory basal nucleus, parvicellular division & LPB & Lateral parabrachial nucleus \\
\hline ABs & Accessory basal nucleus, superficial division & LPO & Lateral preoptic nucleus \\
\hline ac & Anterior commissure & LS & Lateral septum \\
\hline Acb & Accumbens nucleus & LV & Lateral ventricle \\
\hline AD & Anterodorsal nucleus & LY & Lucifer yellow \\
\hline $\mathrm{AHA}$ & Anterior hypothalamic area & $\mathrm{Ma}$ & Mamillary bodies \\
\hline AM & Anteromedial nucleus & MD & Mediodorsal thalamus \\
\hline Amyg & Amygdala & $\mathrm{Me}$ & Medial nucleus of the amygdala \\
\hline Arc & Arcuate nucleus & mlf & Medial longitudinal fasciculus \\
\hline AV & Anteroventral nucleus & MPA & Medial preoptic area \\
\hline B & Basal nucleus of the amygdala & MPB & Medial parabrachial nucleus \\
\hline Bar & Barrington's nucleus & MPO & Medial preoptic nucleus \\
\hline Bmg & Basal nucleus of the amygdala, magnocellular division & MR & Median raphe \\
\hline BNST & Bed nucleus of the stria terminalis & MS & Medial septum \\
\hline Bpc & Basal nucleus of the amygdala, parvicellular division & NLOT & Nucleus of the lateral olfactory tract \\
\hline BSTIA & Intra-amygdaloid division of the BNST & ORX & Orexin \\
\hline CA3 & Field CA3 of the hippocampus & ot & Optic tract \\
\hline CA4 & Field CA4 of the hippocampus & ox & Optic chiasm \\
\hline Cd & Caudate & $\mathrm{Pa}$ & Paraventricular thalamic nucleus \\
\hline Cdc & Central densocellular nucleus & PAC & Periamygdaloid cortex \\
\hline $\mathrm{Ce}$ & Central nucleus of the amygdala & PAG & Periaqueductal gray \\
\hline Cif & Central inferior nucleus & PB & Parabrachial nucleus \\
\hline Cim & Central intermedial nucleus & Pcn & Paracentral nucleus \\
\hline Cla & Claustrum & Pf & Parafascicular nucleus \\
\hline Clc & Central latocellular nucleus & PFA & Perifornical area of the hypothalamus \\
\hline $\mathrm{Cl}$ & Central nucleus of the amygdala, lateral division & $\mathrm{PH}$ & Posterior hypothalamus \\
\hline $\mathrm{Cm}$ & Central nucleus of the amygdala, medial division & Pir & Piriform cortex \\
\hline Co & Core of the nucleus accumbens & PL & Paralaminar nucleus \\
\hline $\mathrm{COa}$ & Anterior cortical nucleus of the amygdala & PM & Premammillary nucleus \\
\hline $\mathrm{CRH}$ & Corticotropin-releasing hormone & PrS & Presubiculum \\
\hline Csl & Central lateral superior nucleus & $\mathrm{Pt}$ & Paratenial nucleus \\
\hline CtB & Cholera toxin B subunit & $\mathrm{Pu}$ & Putamen \\
\hline dIPAG & Dorsolateral periaqueductal gray & $\mathrm{PVH}$ & Paraventricular nucleus of the hypothalamus \\
\hline DM & Dorsomedial hypothalamus & $\mathbf{R}$ & Reticular thalamic nucleus \\
\hline dmPAG & Dorsomedial periaqueductal gray & $\mathrm{Re}$ & Reuniens nucleus \\
\hline DR & Dorsal raphe & $\mathrm{S}$ & Subiculum \\
\hline DRC & Dorsal raphe, caudal division & SCN & Suprachiasmatic nucleus \\
\hline DY & Diamidino yellow & $\operatorname{scp}$ & Superior cerebellar peduncle \\
\hline EC & Entorhinal cortex & $\mathrm{Sh}$ & Shell of the nucleus accumbens \\
\hline EGP & External globus pallidus & sm & Stria medullaris \\
\hline FB & Fast blue & SON & Supraotic nucleus \\
\hline FR & Fluro-ruby & VA & Ventral anterior nucleus \\
\hline f & Fornix & vIPAG & Ventrolateral periaqueductal gray \\
\hline GP & Globus pallidus & VMH & Ventromedial hypothalamus \\
\hline $\mathrm{H}$ & Hippocampus & vsc & Ventral spinocerebellar tract \\
\hline
\end{tabular}




\section{Injections}

Prior to surgery, stereotaxic coordinates for each injection site were specified for each animal from a magnetic resonance imaging (MRI) scan. Animals were anesthetized (see below) and placed into an MRI-compatible stereotaxic frame. Scans of the head were performed with a 1.5T or 3T scanner with a receive-only coil placed over the top of the head, producing a 3D magnetization-prepared rapid acquisition gradient echo image of the brain in stereotaxic alignment $\left(T_{1}\right.$ weighted, resolution of $0.7-0.8 \mathrm{~mm}$ voxels). Distance from a line through the ear bars was calculated for each injection site using the MR images, with reference to the atlas of Szabo and Cowan (1984).

For both surgery and MRI, anesthesia was induced with ketamine $(10 \mathrm{mg} / \mathrm{kg}$, i.m.) and xylazine $(0.67 \mathrm{mg} / \mathrm{kg}$, i.m.) and maintained with a gaseous mixture of oxygen, nitrous oxide, and either halothane or isoflurane throughout the procedure. A long-lasting analgesic (buprenorphine, 0.01-0.03 mg/kg, i.m.) was given immediately following surgery and every 8-12 hours for 2-3 days. An antibiotic (cefazolin, $25 \mathrm{mg} / \mathrm{kg}$, i.m.) was given twice a day for 5-7 days.

Surgery was performed under full sterile precautions. Animals were placed in a stereotaxic frame, the skull was exposed, and burr holes were made at the injection site coordinates. Electrophysiological recordings were done to refine and confirm coordinates determined by MRI. A tungsten electrode was lowered into the expected injection track to record spontaneous, multiunit activity along the depth. Differences in activity indicated cortex/white matter boundaries, sulci positions, and the top and bottom of the brain. For recording and injecting into the midline thalamus, the sagittal sinus was retracted a few millimeters to one side to expose the midline.

Retrograde tracers injected into each animal included fast blue (FB, Sigma, St. Louis, MO), diamidino yellow (DY, Sigma), tetramethylrhodamine (fluro-ruby, FR, 10,000 MW, Molecular Probes, Eugene, OR), lucifer yellow (LY, 10,000 MW, Molecular Probes), and cholera toxin B subunit (CtB, List Biological, San Jose, CA) or cholera toxin B subunit conjugated to colloidal gold conjugate (CtB-gold, List Biological). In addition, injections were made with the anterograde tracer biotincoupled dextran amine (BDA, 10,000 MW, Molecular Probes). Tracers were injected through a micropipette using an air pressure system that applied 25-msec air pulses to the pipette. The injection volumes were calculated as the crosssectional area of the pipette times the distance traveled by the meniscus, which was marked on the pipette. Movement of the meniscus during the injections was monitored using a dissecting microscope. Tracers were injected at a volume of 0.1-1.3 $\mu \mathrm{L}$, depending on tracer sensitivity and the size of the target area. To avoid the spread of tracer along pipette track, the pipette was left in place for $\mathbf{3 0}$ minutes after each injection. This resulted in little spread of tracer into white matter. Six different tracers were injected into each animal.

\section{Perfusion and tissue processing}

Following survival times of about 2 weeks, animals were deeply anesthetized with ketamine $(10 \mathrm{mg} / \mathrm{kg}$, i.m.) followed by sodium pentobarbital (25-30 $\mathrm{mg} / \mathrm{kg}$ i.v.). Animals were perfused transcardially with heparinized saline, followed by a sequence of $4 \%$ paraformaldehyde solutions buffered at $\mathrm{pH}$ $6.5, \mathrm{pH} 9.5$, and finally at $\mathrm{pH} 9.5$ with $10 \%$ sucrose (Carmichael and Price, 1994). The brain was removed from the skull, blocked, placed in $4 \%$ paraformaldehyde with $10 \%$ sucrose, and then in phosphate-buffered $20 \%$ and $30 \%$ sucrose at $4{ }^{\circ} \mathrm{C}$ until it sank (usually 3 days).

Brains were frozen in isopentane with dry ice and cut with a freezing microtome into 10 collated series of $50-\mu \mathrm{m}$ coronal sections. One series was processed for each tracer. For fluorescent tracers FB and DY, sections were mounted without staining and examined with fluorescence microscopy. FR, LY, and CtB were processed immunohistochemically with an avidin-biotin-horseradish peroxidase technique; BDA was processed with the avidin-biotin-peroxidase method (Carmichael and Price, 1994). Additional series were stained for Nissl. Several sections were used to optimize immunohistochemical staining for 5-HT, ORX, and CRH (see below) using an avidin-biotin-horseradish peroxidase technique (Carmichael and Price, 1994). CtB-gold injected into Pa was revealed with silver intensification (Kritzer and Goldman-Rakic, 1995), and the series was counterstained with Nissl stain. Separate series of sections from this brain were stained for 5-HT, ORX, and CRH followed by silver intensification to reveal double-labeled neurons.

The polyclonal 5-HT antibody was raised in rabbit against 5-HT coupled to BSA with paraformaldehyde (ImmunoStar, Hudson, WI) and has been reported previously for immunohistochemical staining in M. fascicularis (e.g., LaMotte, 1988; Westlund et al., 1990; Moore and Speh, 2004). Sections incubated ( 48 hours at $4^{\circ} \mathrm{C}$ ) in a series of nine dilutions of the primary antibody from $1: 20,000$ to $1: 50,000,000$ showed decreased staining until staining was no longer present. A sample of sections incubated without the primary antiserum did not exhibit any immunoreactivity. In addition, a sample of sections was exposed to $1: 10,000$ dilution of primary antiserum which had been preadsorbed for 24 hours at $4^{\circ} \mathrm{C}$ with serotonin BSA conjugate (ImmunoStar) at a concentration of $10 \mu \mathrm{g} / \mathrm{mL}$ and $90 \mu \mathrm{g} / \mathrm{mL}$. These sections did not exhibit any immunoreactivity. A dilution of $1: 1,000,000$ was optimal for visualizing axons, and a dilution of $1: 5,000,000$ was optimal for visualizing neurons double-labeled with CtB-gold (see below).

The polyclonal ORX-B antibody was raised in goat against a 19 mer peptide (amino acids 78-96) mapping at the C-terminus of human ORX-B (Santa Cruz Biotechnology, Santa Cruz, CA). This antiserum was previously shown to label hypothalamic neurons in sections from wildtype mice but not in sections from ORX knockout mice (Crocker et al., 2005), demonstrating its specificity to the ORX epitope. A dilution of the primary antiserum at 1:10,000 incubated with sections for 48 hours at $4^{\circ} \mathrm{C}$ was found to be optimal for both axons and neurons, whereas $10 \times$ and $100 \times$ of this dilution and a sample of sections incubated without the primary antiserum did not produce any labeling. In addition, a sample of sections was exposed to $1: 10,000$ dilution of the primary antiserum that had been preadsorbed for 24 hours at $4^{\circ} \mathrm{C}$ with ORX-B (Santa Cruz) at a concentration of $0.4 \mu \mathrm{g} / \mathrm{mL}, 4 \mu \mathrm{g} / \mathrm{mL}$, and $40 \mu \mathrm{g} / \mathrm{mL}$. No labeling was observed following incubation with the highest concentration of peptide. The location of ORX labeling in the hypothalamus in the present study corresponds to that which was previously demonstrated in monkeys with a different antibody (Horvath et al., 1999).

The polyclonal $\mathrm{CRH}$ antibody was raised in rabbit against rat/human $\mathrm{CRH}$ and had been preadsorbed with melanocyte 
stimulating hormone and human alpha globulins (generously provided by W. Vale and J. Rivier, Salk Institute, La Jolla, CA). This antiserum (lot rc70) has been previously characterized in M. fascicularis (Cha and Foote, 1988; Foote and Cha, 1988). It has been shown that staining with the antiserum is abolished by preadsorption with $10 \mathrm{nmol} / \mathrm{mL}$ of rat/human $\mathrm{CRH}_{1-41}$, but not with alpha-melanocyte stimulating hormone or porcine peptide histidine-isoleucine ${ }_{1-27}$ (Bassett and Foote, 1992). Sections incubated ( 48 hours at $4^{\circ} \mathrm{C}$ ) in a series of seven dilutions of the primary antibody from $1: 2,000$ to $1: 2,000,000$ showed decreased staining until staining was no longer present. A sample of sections incubated without the primary antiserum did not exhibit any immunoreactivity. A dilution of $1: 100,000$ was found to be optimal for both axons and neurons.

\section{Analysis}

Mounted sections were examined with light/fluorescence microscopy. Injection sites, labeled neurons, and labeled axonal varicosities were manually plotted using a microscope digitizer system (AccuStage, Shoreview, MN) interfaced with a personal computer. Each labeled neuron and labeled axonal varicosity was plotted as a single point. In order to ensure that all cells or varicosities were counted, and were not double counted, they were mapped in successive $200-\mu \mathrm{m}$-wide linear traverses across the section; because of this the cells or varicosities often appear to be in linear rows on the maps. The resolution of the maps along each traverse is $5 \mu \mathrm{m}$. Thalamic nuclei and other structures were drawn onto printed plots using camera lucida methods and adjacent sections stained for Nissl, ACHE, or myelin. The terminology used for the midline and intralaminar nuclei was adopted from The Thalamus of the Macaca mulatta by Olszewski (1952) with a few modifications (Hsu and Price, 2007).

Photomicrographs were captured with a Nikon DXM 1200 digital camera connected to a PC with ACT-1 image capture software. The images were cropped and adjusted for brightness, contrast, and sharpness when necessary using Adobe Photoshop CS (San Jose, CA).

\section{RESULTS \\ Pa outputs}

Anterograde tracer injection into the dorsal midline thalamus. The anterograde tracer BDA was injected into the dorsal midline thalamus in case OM67 (Fig. 1A). Most of the injection was centered in $\mathrm{Pa}$ and paratenial nucleus $(\mathrm{Pt})$, although tracer also spread into the rostral part of the central densocellular nucleus (Cdc), and a small dorsal portion of the anteromedial nucleus (AM). In the previous study of cortical connections of $\mathrm{Pa}$ and $\mathrm{Pt}$, this injection was shown to label axons in cortical areas within the medial prefrontal network (Hsu and Price, 2007); many of the subcortical areas with labeling are also specifically connected to the medial network.

Thus, high densities of labeled axons and axonal varicosities were found in the ventral striatum, especially in the accumbens nucleus and rostral-medial caudate nucleus (Figs. $1 C, D, 2 A-C)$. The heaviest labeling was in the shell compared to the core of the accumbens. The accumbens nucleus is strongly related to the medial prefrontal network, and the shell is particularly specifically connected to area 25 (Ferry et al., 2000), which is strongly interconnected with $\mathrm{Pa}$ (Hsu and
Price, 2007). The more central part of the striatum, which is connected to the orbital prefrontal network (Ferry et al., 2000), has many fewer labeled axons from $\mathrm{Pa}$ and $\mathrm{Pt}$.

There were also many labeled axons and varicosities in the amygdala, which is itself strongly interconnected with both the accumbens nucleus and the medial prefrontal network (Price, 2003). The highest concentration of labeled axonal varicosities was found in the magnocellular and parvicellular divisions of the basal nucleus (Fig. 1F), and in the intraamygdaloid division of the bed nucleus of the stria terminalis (BNST) (Fig. 1G). Smaller numbers of varicosities are also found in most other nuclei of the amygdala, including the accessory basal nucleus (especially the lateral edge that borders the basal nucleus), the medial and lateral parts of the central nucleus, and the anterior cortical and medial nuclei. Relatively few labeled varicosities were present in the medial part of the accessory basal nucleus and the lateral region of the lateral nucleus.

There were also a number of labeled varicosities in the anterior amygdaloid area, the extended amygdala and BNST proper (Figs. 1E, 3B). Labeled varicosities are found in all divisions of BNST, but they are most dense in the dorsal, anterior part, above the anterior commissure. The density of labeled varicosities decreases in more caudal and ventral parts. There are also labeled varicosities in adjacent regions of the caudate nucleus, dorsolateral to BNST, and the anterior hypothalamus, ventral to BNST, such that there are no sharp boundaries in the distribution of the label.

Dense labeling was also found in the deep layers of the entorhinal cortex. (Fig. 1D,E). This is most dense in the rostral and to a lesser extent the rostral lateral divisions. The labeling decreases markedly in the more caudal divisions of the entorhinal cortex.

In the hypothalamus there was substantial axonal label in the medial preoptic area, suprachiasmatic nucleus, and other parts of the medial hypothalamus, although the label is not concentrated in any of these nuclei (Figs. 1E, 3C, 4A,B). There was also substantial label in the lateral hypothalamus (Figs.

Figure 1.

Case OM67. Anterograde tracer BDA injected into the dorsal midline thalamus (A), and the resulting labeling in several subcortical structures (rostral-caudal, B-H). Labeled varicosities are represented by transparent gray dots, with areas of overlay appearing darker. The accumbens nucleus contained the highest density of labeled axons and axonal varicosities, particularly in the shell compartment (C,D). Dashed lines show approximate delineation of core and shell based on our observations and previous reports (Meredith et al., 1996; Brauer et al., 2000). High numbers of labeled varicosities were found in the head of the caudate (B), and extended rostrocaudally along the dorsal part of the caudate (C-G). In the amygdala, dense labeling was found in both the magnocellular and parvicellular divisions of the basal nucleus $(E, F)$, as well as in the bed nucleus of the stria terminalis $(E)$, and its intra-amygdaloid division (G). Dense labeling was found in the hypothalamus, particularly in the medial preoptic area (E). In addition to previously reported labeling in prefrontal cortical areas, dense labeling was also found in the entorhinal cortex $(D, E)$. In the midbrain, moderate labeling was found in the dorsomedial and dorsolateral periaqueductal gray $(\mathrm{H})$. In this and other figures, the apparent distribution of labeled varicosities or neurons in linear rows is due to mapping of the section in 200- $\mu \mathrm{m}$-wide linear traverses, in order to ensure even mapping of all the labeling. The dashed lines show layer IV or the border between layers III and V in the temporal cortex. Scale bars $=1 \mathrm{~mm}$ in $\mathrm{A} ; 5 \mathrm{~mm}$ in $\mathrm{B}-\mathrm{H}$. 


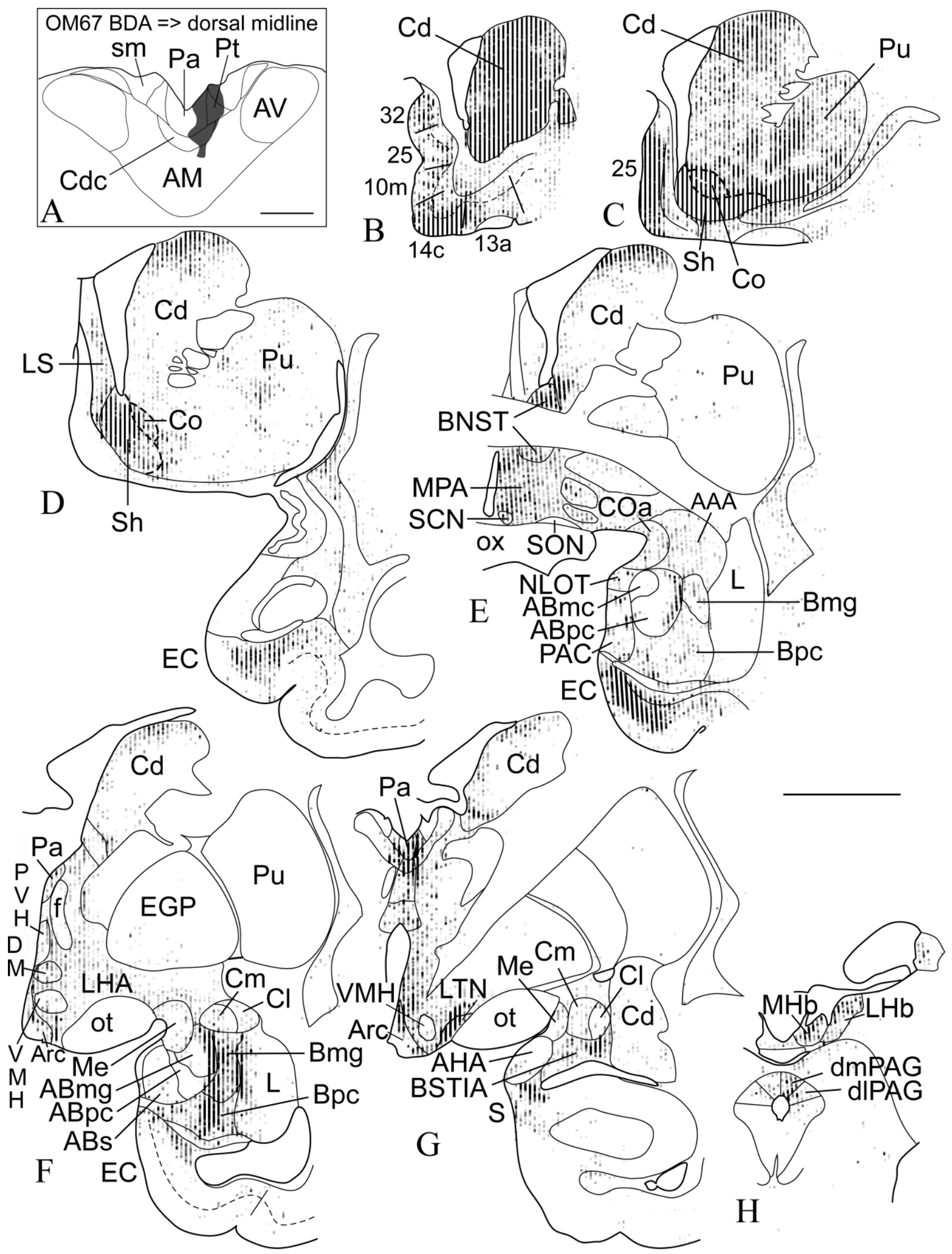

Figure 1 
3D, 4C,D). Few axons or axonal varicosities were observed within cell body regions of the paraventricular and supraoptic nuclei of the hypothalamus, although labeled axons were
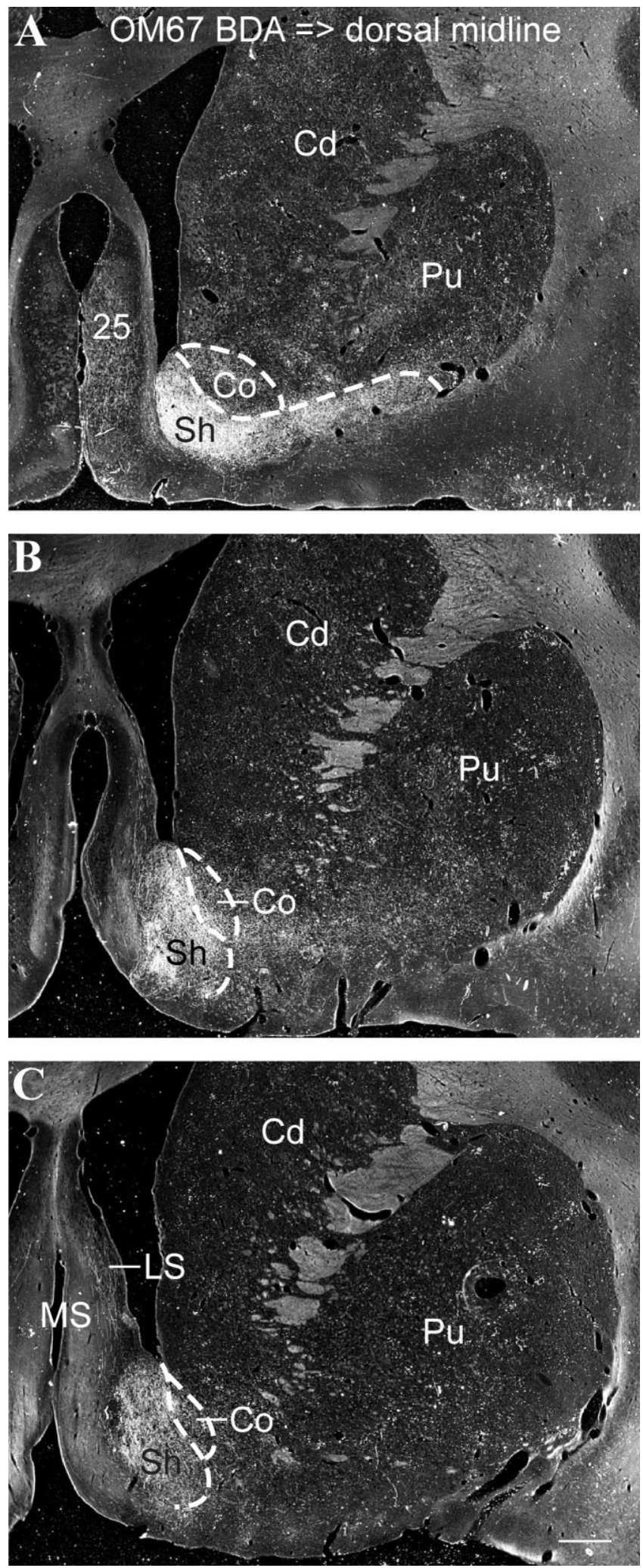

found immediately lateral or dorsal to the nuclei (Figs. 1F, 3D, 4B,D). Electron microscope studies have shown that the dendrites of paraventricular and supraoptic nuclei neurons extend laterally and dorsally from the nuclei such that the dendritic receptive zone of the nucleus is outside the zone of cell bodies (Silverman and Oldfield, 1984; Oldfield and Silverman, 1985). The axons therefore pass through the dendrites of the paraventricular and supraoptic nuclei, and may synapse on them. Rostral to the hypothalamus, moderate labeling was also found in the lateral septum (Figs. 1D, 2B,C, 3A). Dense labeling in prefrontal cortical areas, including area 25 (Figs. 1C, 2A), was described in a previous study (Hsu and Price, 2007).

Retrograde tracer injections into the striatum, amygdala, entorhinal cortex, lateral septum, and hypothalamus. Several experiments were analyzed in relation to label in the midline thalamus that had been prepared for previous studies in which retrograde axonal tracers were injected in structures shown above to receive projections from the dorsal midline thalamus. In these cases, labeled neurons were found in $\mathrm{Pa}$ and/or Pt, as well as in other midline thalamic nuclei (Table 1). These confirm most of the projections described above from anterograde tracer experiments, and show that they arise primarily from $\mathrm{Pa}$, and in some cases also from $\mathrm{Pt}$ and other midline nuclei.

Striatum. Two different retrograde tracers each were injected into different parts of the striatum in cases OM39 (Table 1), OM40 (Fig. 5A,F; Table 1), and OM43 (Fig. 5K,P; Table 1). Case OM46 had one injection in the dorsal-medial caudate (Fig. $5 \mathrm{U}$; Table 1).

Injections into the accumbens nucleus in OM40 (FB) and OM43 (FB) resulted in a high density of labeled neurons in $\mathrm{Pa}$ and in some cases in Pt and other midline nuclei (Fig. 5B-E, LO). The labeling in the Pt was especially marked in OM40 (Fig. $5 A-E)$, from an FB injection that involved both the core and shell region of the accumbens nucleus. There was somewhat less label in Pt in OM43, where the injection of FB was centered in the shell of the accumbens nucleus. The labeling was continuous through the rostral-caudal extent of $\mathrm{Pa}$. Lesser concentrations of labeled neurons were found in the $\mathrm{Cdc}$ and reuniens (Re) in OM40 (Fig. 5D), and in the Cdc, Re, central latocellular (Clc), central intermedial (Cim), paracentral (Pcn), and parafascicular nucleus (Pf) in OM43 (Fig. 5L-O). Previously, these same injections were shown to produce labeled neurons primarily in cortical areas of the medial prefrontal network (Ferry et al., 2000).

By comparison, retrograde tracer injections into central parts of the putamen and caudate nucleus, which are primarily connected to the orbital prefrontal network or other regions of cortex, resulted in relatively little labeling in $\mathrm{Pa}$ or $\mathrm{Pt}$. An

Figure 2.

Case OM67. Darkfield photomicrographs of the striatum following a BDA injection into the dorsal midline thalamus (Fig. 1A), at three rostral-caudal levels (A-C). Labeled axons and axonal varicosities were densely concentrated in ventromedial striatum, in the shell of the accumbens nucleus. Dashed lines show approximate delineation of core and shell based on our observations and previous reports (Meredith et al., 1996; Brauer et al., 2000). Labeling can also be seen in cortical area 25 (A) and lateral septum (C) (see also Fig. 1C,D). Scale bar $=1 \mathrm{~mm}$. 

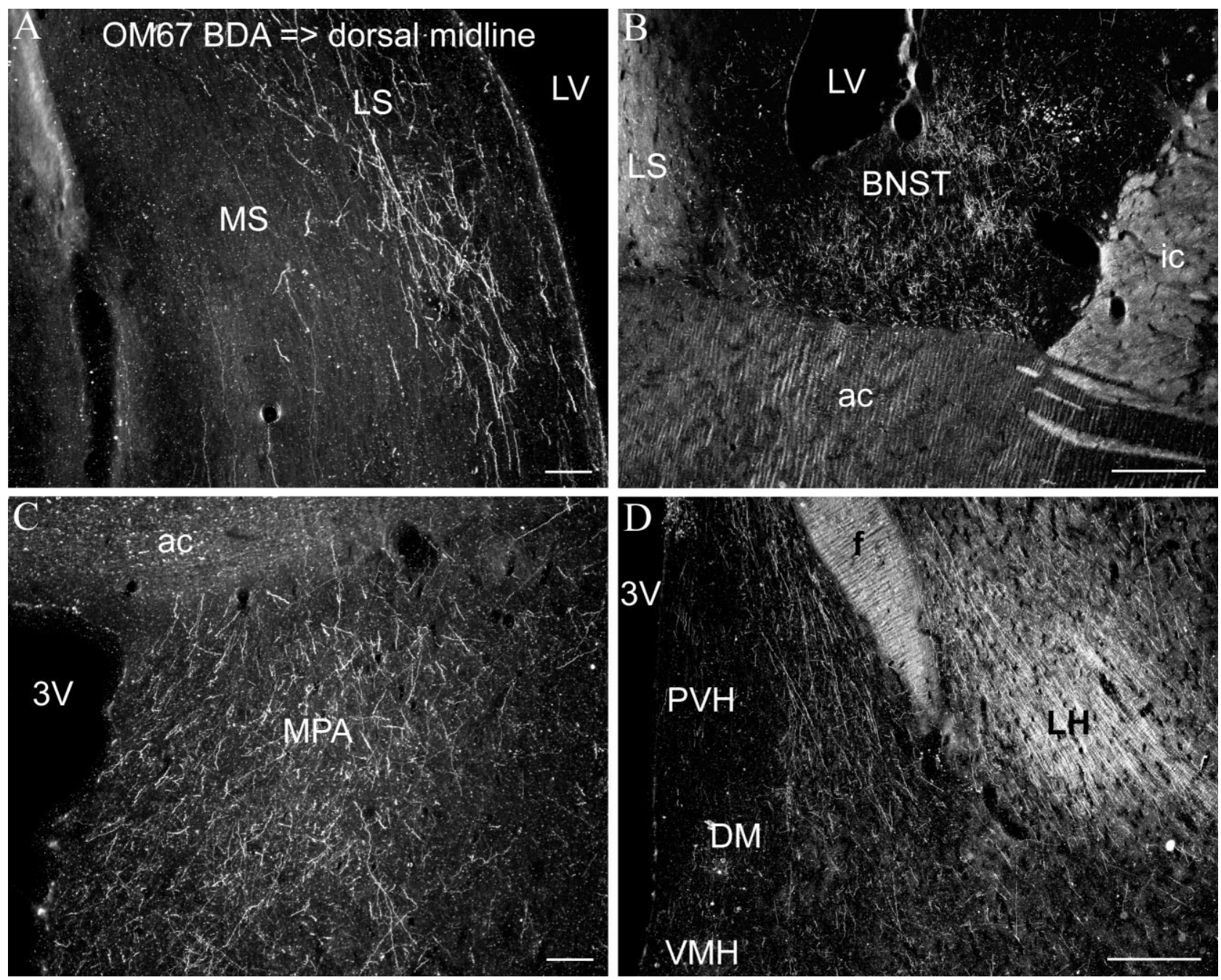

Figure 3.

Case OM67. Darkfield photomicrographs showing the distribution of labeled axons and axonal varicosities following an anterograde tracer (BDA) injection into the dorsal midline thalamus (Fig. 1A) in the lateral septum (A), bed nucleus of the stria terminalis (B), medial preoptic area of the hypothalamus (C), and lateral hypothalamus (D). Scale bars $=100 \mu \mathrm{m}$ in A,C; $500 \mu \mathrm{m}$ in B,D.

injection of DY into the medial putamen in OM40, at the border of the accumbens nucleus, labeled neurons mostly in the Cdc with a few labeled neurons in the ventral parts of $\mathrm{Pa}$ (Fig. $5 \mathrm{~F}-\mathrm{J})$. Labeled neurons were also found in the Pf (Fig. 5J). An injection of CtB into the central part of the putamen in OM43 resulted in high numbers of labeled neurons in the Cdc, Cim, and central inferior nucleus (Cif) (Fig. 5P-T). Both of these injections were previously found to produce labeled neurons primarily in cortical areas of the orbital prefrontal network (Ferry et al., 2000). With both injections in the putamen, labeled neurons were also observed in the central lateral superior nucleus (Csl) (Fig. 5I,J,S,T). An injection of FB into the dorsal-medial aspect of the caudate nucleus in OM46 labeled ventral parts of $\mathrm{Pa}$ as well as Csl, but not in $\mathrm{Pt}$ (Fig. $5 \mathrm{U}-\mathrm{Y}$ ). This injection involved both the medial and central parts of the caudate nucleus; it was previously shown to label cortical neurons in areas related to both the medial and orbital prefrontal networks, as well as in the dorsolateral prefrontal cor- tex (Ferry et al., 2000). A smaller injection of FB in the medial caudate in OM39 produced a similar pattern of label in $\mathrm{Pa}$, with little or no label in $\mathrm{Pt}$, while an injection of $\mathrm{DY}$ in the lateral caudate in the same case labeled virtually no neurons in the midline nuclei (not illustrated, Table 1).

Taken together, these cases confirm the anterograde data (OM67, above) that $\mathrm{Pa}$ projects strongly to the accumbens nucleus (especially the shell) and the rostromedial caudate nucleus. Pt also projects to the accumbens nucleus, but more to the core than the shell.

Amygdala and entorhinal cortex. An injection of FR in OM73 was centered in the magnocellular basal nucleus of the amygdala, with involvement of the accessory basal and lateral nuclei. This experiment had substantial numbers of retrogradely labeled neurons in $\mathrm{Pa}, \mathrm{Pt}$, and parts of the Cdc (Fig. 6A-E; Table 1). Lower numbers of labeled neurons were seen in the Clc, Re, Pcn, and Pf. A similar, but smaller injection of CtB into the amygdala in OM58 produced a similar pattern of 

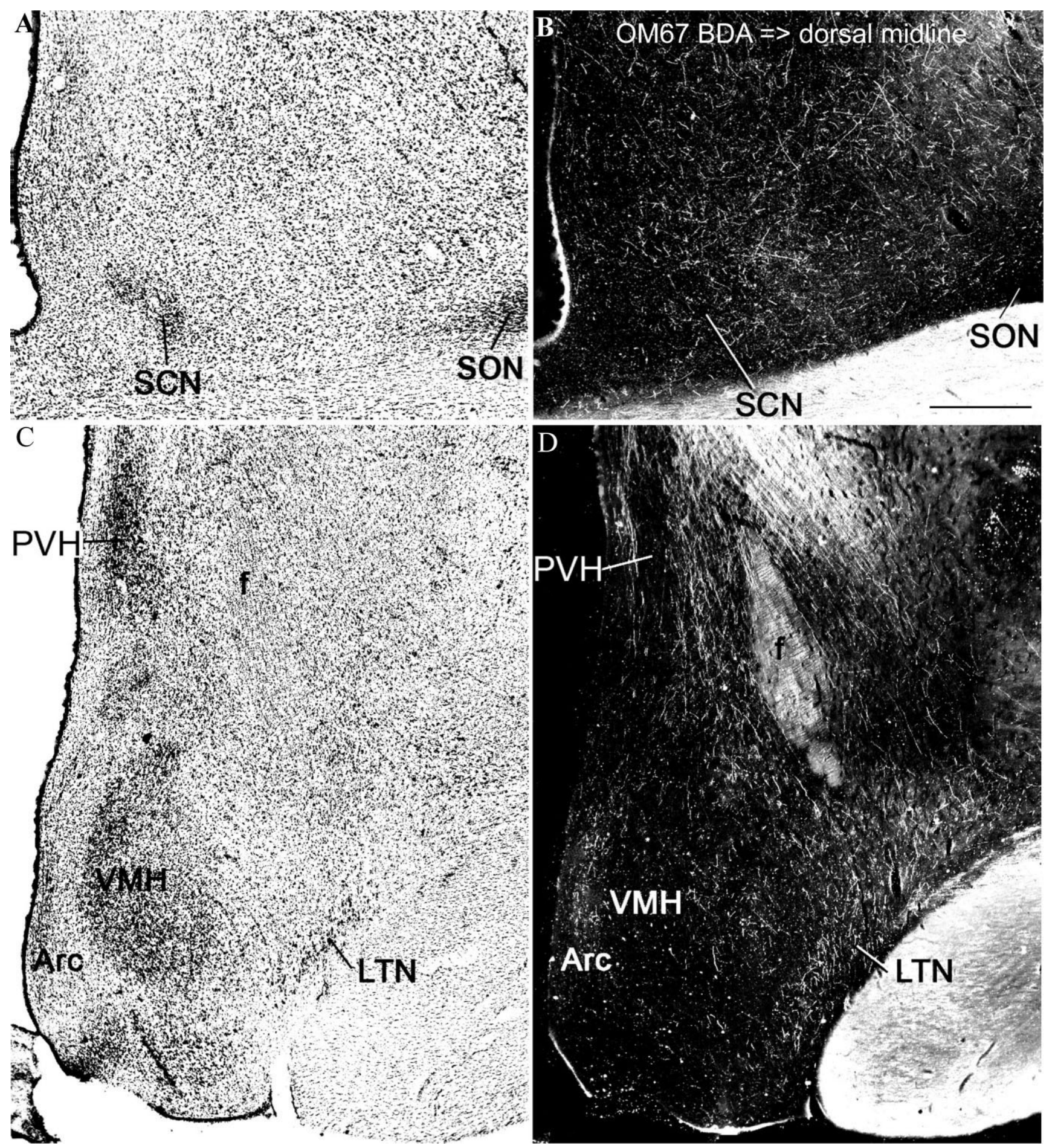

Figure 4.

Case OM67. A,B: Brightfield and darkfield photographs from a section of the anterior hypothalamus showing the suprachiasmatic and supraoptic nuclei (in A), and the distribution of labeled axons and axonal varicosities (in B) following injection of the anterograde tracer BDA into the dorsal midline thalamus (see Fig. 1A). Note that the labeled axons are found over the suprachiasmatic nucleus and around the supraoptic nucleus, but are not restricted to them. C,D: Brightfield and darkfield photographs from a section of the tuberal hypothalamus, showing the paraventricular, ventromedial, arcuate, and lateral tuberal nuclei in relation to the labeled axons from the dorsal midline thalamus. Scale bar = $500 \mu \mathrm{m}$. 
TABLE 1. Relative Densities of Labeled Axons or Neurons in the Midline Thalamus Following Injections in Subcortical Structures

\begin{tabular}{|c|c|c|c|c|c|c|c|c|}
\hline \multirow[b]{2}{*}{ Case No. } & \multirow[b]{2}{*}{ Area Injected } & \multirow[b]{2}{*}{ Tracer } & \multicolumn{6}{|c|}{ Labeled Axons or Neurons in: } \\
\hline & & & $\mathrm{Pa}$ & $\mathrm{Pt}$ & Cdc & Csl & Cim & $\mathrm{Re}$ \\
\hline \multicolumn{9}{|c|}{ Anterograde Tracers } \\
\hline DM28 & Amyg Ce/BSTIA & ${ }^{3} \mathrm{H}$-Leu & +++ & - & ++ & - & - & - \\
\hline OM3 & Amyg Ant Cort Nuc & ${ }^{3} \mathrm{H}-$ Leu & ++ & + & - & - & + & - \\
\hline OM73 & Amyg B/AB/L & FR & +++ & ++ & + & + & + & ++ \\
\hline OM43 & Med. Hypothalamus & BDA & +++ & - & + & + & + & + \\
\hline OM44 & Med. Hypothalamus & BDA & +++ & - & ++ & ++ & ++ & + \\
\hline OM46 & Med. Hypothalamus & BDA & +++ & ++ & + & + & + & - \\
\hline \multicolumn{9}{|c|}{ Retrograde Tracers } \\
\hline OM40 & Accumbens & FB & +++ & +++ & + & + & & + \\
\hline OM43 & Accumbens Shell & FB & +++ & + & + & $+/-$ & + & + \\
\hline OM39 & Rostromedial Caudate & FB & +++ & $+/-$ & ++ & - & - & + \\
\hline OM46 & Medial Caudate & FB & ++ & $+/-$ & + & ++ & - & - \\
\hline OM40 & Ventral Putamen & DY & $+/-$ & - & +++ & ++ & + & + \\
\hline OM43 & Putamen & CtB & - & - & +++ & ++ & + & + \\
\hline ОМ39 & Rostrolateral Caudate & DY & - & - & - & - & - & - \\
\hline OM16 & Lateral Septum & DY & +++ & + & - & - & - & - \\
\hline OM37 & Ant Hypothal/MPOA & FB & ++ & $-1+$ & - & - & - & - \\
\hline OM38 & Lat Mid-Hypothal & FB & ++ & + & - & - & - & ++ \\
\hline OM58 & Amyg B/L & CtB & +++ & + & ++ & + & + & ++ \\
\hline OM73 & Amyg B/AB/L & FR & +++ & ++ & ++ & + & + & + \\
\hline OM78 & Entorhinal Ctx & LY & + & ++ & ++ & + & ? & ++ \\
\hline OM35 & PAGvl & FB & + & - & - & - & - & - \\
\hline OM36 & PAGI & FB & + & - & - & - & - & - \\
\hline OM37 & PAGdI & CtB & + & - & - & - & - & - \\
\hline OM35 & Lateral to PAG & CtB & - & - & - & - & - & - \\
\hline OM36 & Lateral to PAG & CtB & - & - & - & - & - & - \\
\hline
\end{tabular}

For abbreviations, see list.

labeled neurons (Fig. 7; Table 1). In OM78, an injection of LY was made in the entorhinal cortex. Labeled neurons were found in this experiment in the Pt, with few labeled neurons in $\mathrm{Pa}$ (Fig. 6F-J). Labeled neurons were also found in the Clc, $\mathrm{Re}$, and Cdc. These experiments confirm projections from $\mathrm{Pa}, \mathrm{Pt}$, and other midline nuclei to the amygdala. The projection to the entorhinal cortex, in contrast, may arise primarily from the $\mathrm{Pt}$ and other midline nuclei outside $\mathrm{Pa}$.

Septum and hypothalamus. The lateral septum was injected with DY in OM16. In this case there were many labeled neurons in $\mathrm{Pa}$, with fewer neurons in Pt (Fig. 6K-O; Table 1). Experiments with injections in the hypothalamus, including OM37, with an injection of FB into the anterior hypothalamus and medial preoptic area at the level of the paraventricular hypothalamic nucleus, supraoptic nucleus, and suprachiasmatic nucleus (Fig. 6P), and OM38 with an injection in the posterior lateral hypothalamus at the level of the premammillary nuclei (Fig. 6U), contained labeled neurons mostly in Pa. With the more anterior injection (OM37), only a few labeled neurons were found outside $\mathrm{Pa}$ (Fig. 6R-T). The more posterior injection (OM38) produced labeled neurons in the Re and the posterior part of $\mathrm{Pt}$, in addition to those found throughout $\mathrm{Pa}$ (Fig. 6V-Y).

Periaqueductal gray (PAG). Three experiments are available with injections in the PAG (OM35 FB, OM36 FB, OM37 CtB), and two with injections lateral to the PAG (OM35 CtB, OM36 CtB). All three injections into the PAG produced retrogradely labeled neurons in $\mathrm{Pa}$, but few if any in other midline thalamic nuclei (Table 1). The injections into the midbrain lateral to the PAG did not result in labeled neurons in $\mathrm{Pa}$.

\section{Inputs to $\mathbf{P a}$}

Retrograde tracer injections into the dorsal midline. An injection of CtB was made into the dorsal midline in case OM64, involving $\mathrm{Pa}$ and $\mathrm{Pt}$ (Fig. 8A). Labeled subcortical neu- rons were found mostly in three regions: the amygdala and hippocampal formation, the septum and hypothalamus, and the dorsal raphe and PAG.

Labeled neurons are widely distributed in the septum and especially in the medial hypothalamus, including the suprachiasmatic nucleus (Fig. $8 \mathrm{C}$ ), medial preoptic area (Fig. $8 \mathrm{C}$ ), ventromedial nucleus (Fig. 8D), arcuate nucleus (Fig. 8D,E), and perifornical area (Fig. 8E). The neurons are not specifically concentrated in any of the nuclei, but extend relatively diffusely through the region.

Within the amygdala, many labeled neurons were concentrated in the ventral, parvocellular part of the basal nucleus, and there were scattered neurons in other nuclei, especially the anterior cortical amygdaloid nucleus. In the hippocampal formation, dense concentrations of neurons were labeled in the presubiculum (Fig. 8D-F). Moderate numbers of neurons were labeled in the entorhinal cortex and in the CA3/4 region (hilus of the dentate gyrus) (Fig. 8E-H).

In the midbrain, dense concentrations of labeled neurons were found in the dorsal raphe, particularly in the caudal division (Fig. 9). There were also labeled neurons scattered through the median raphe. More caudally, a few neurons are labeled in the rostral part of the locus ceruleus.

Neurons are labeled in all columns of PAG, with a slight concentration in the dorsolateral column (Fig. 9A-E). In the parabrachial nucleus, labeled neurons are scattered through all of the subnuclei in the lateral part of the nucleus, without apparent concentration in any given subnucleus (Fig. 9E-G). A smaller number of labeled neurons is found in the medial parabrachial nucleus.

A slightly larger injection of CtB-gold was made into the dorsal midline thalamus in OM78. Like OM64, this case had retrogradely labeled neurons in the medial and lateral hypothalamus, amygdala, subiculum, PAG, dorsal/median raphe nuclei, and the parabrachial nuclei (Figs. 12, 13). 

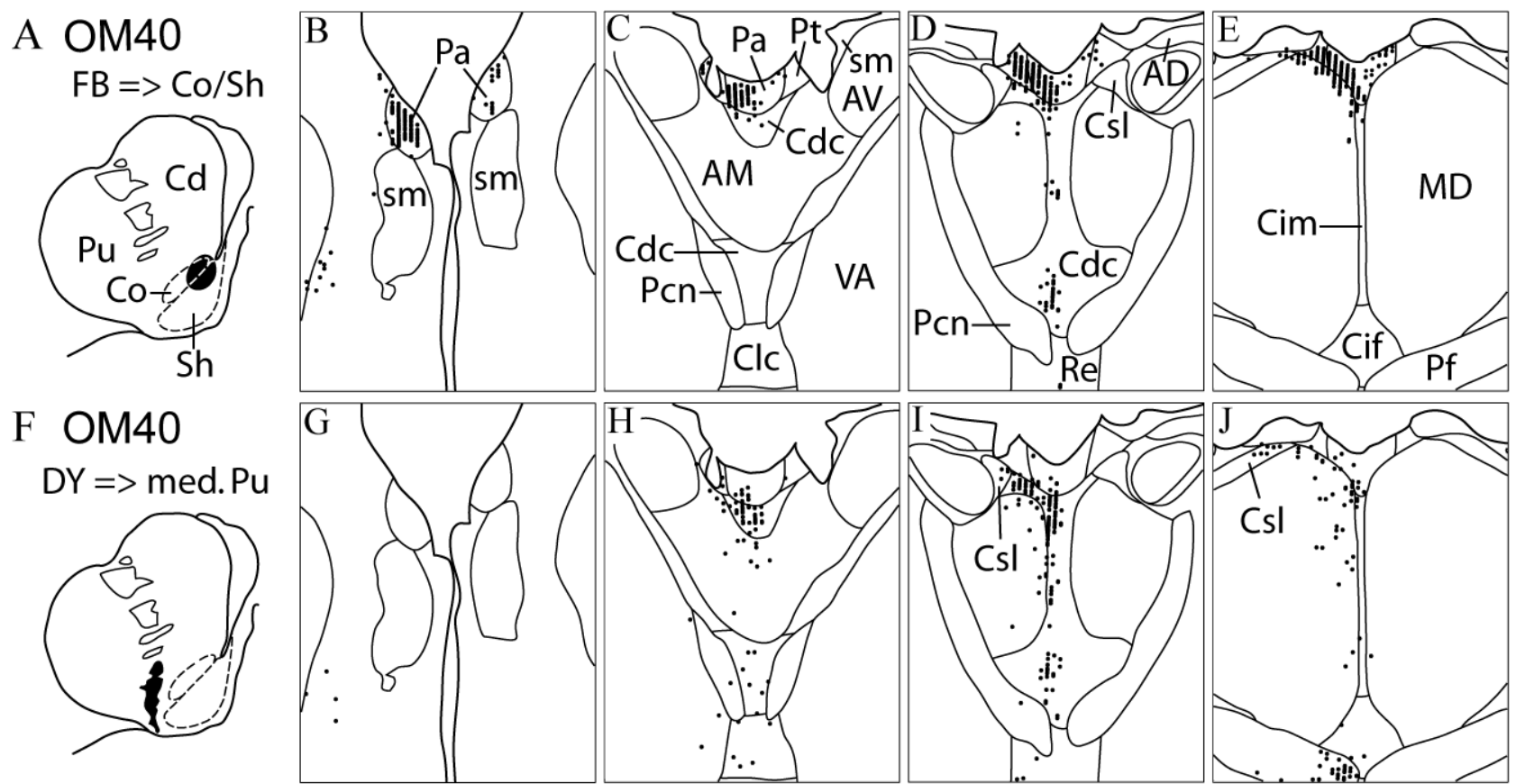

K OM43

$\mathrm{FB}=>\mathrm{Sh}$
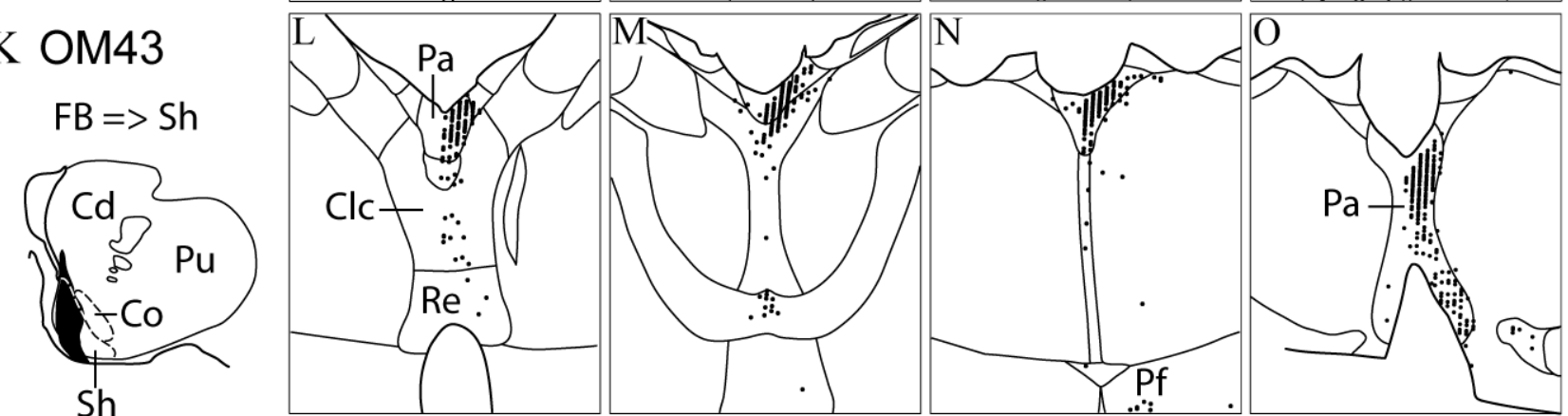

P OM43

$\mathrm{CtB}=>$ lat. $\mathrm{Pu}$
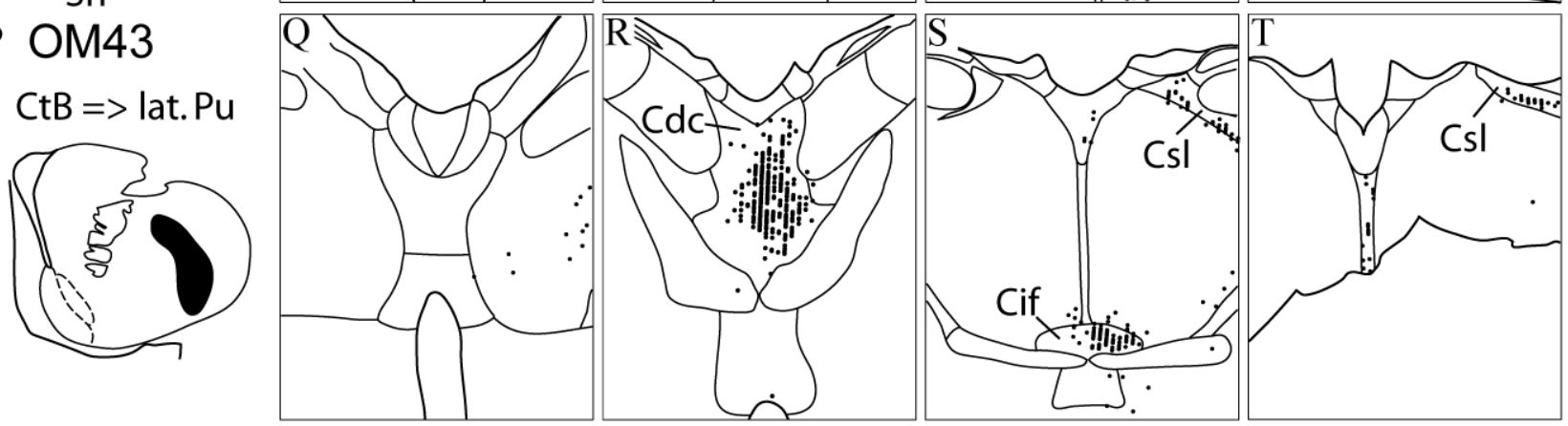

U OM46
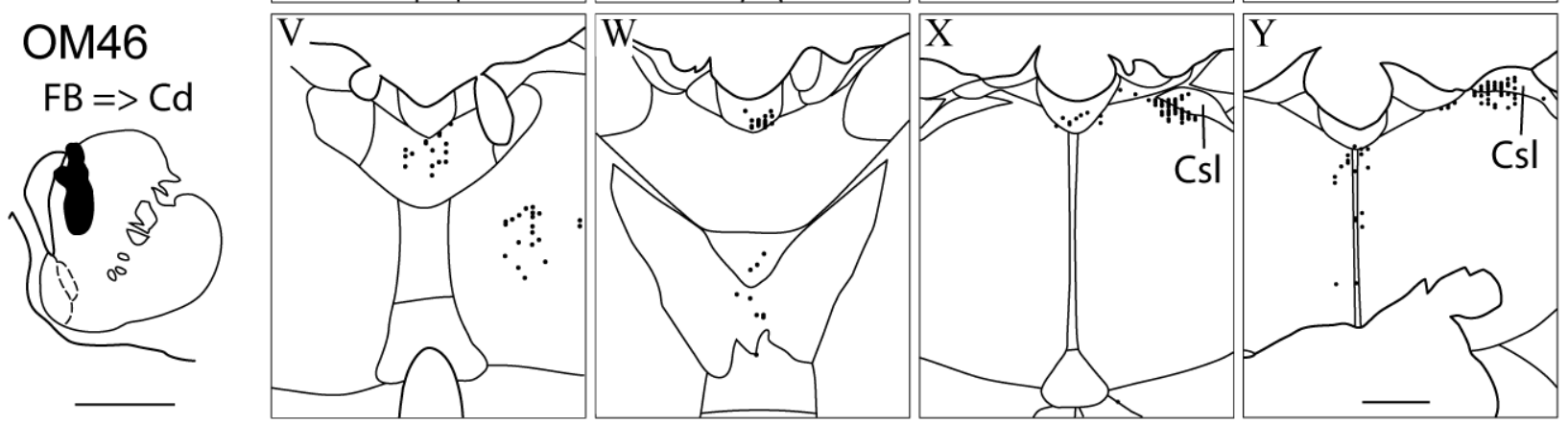

Figure 5 
Anterograde tracer injections into the amygdala and hypothalamus. Some of the afferent projections to the midline thalamus shown in the OM64 and OM78 were confirmed by other experiments with injections of anterograde tracers into the amygdala and medial hypothalamus. These also show that the projections terminate primarily in $\mathrm{Pa}$, with lighter projections to other midline nuclei.

Three experiments were analyzed in which injections of anterograde tracers were made in different nuclei of the amygdala (Table 1). OM3 had a small injection of ${ }^{3} \mathrm{H}$-leucine into the anterior cortical nucleus of the amygdala. It shows good axonal label in $\mathrm{Pa}$, with lower density of label in $\mathrm{Pt}$ and Cim (Table 1). In DM28, an injection of ${ }^{3} \mathrm{H}$-leucine was made into the caudal-dorsal part of the amygdala, involving the central nucleus and the intra-amygdaloid part of the bed nucleus of the stria terminalis. There is dense autoradiographic axonal label in the posterior part of $\mathrm{Pa}$ (Fig. 10B), with less labeling in more anterior parts of the nucleus. Label is also found in Cdc, but little or no label in other midline or intralaminar nuclei (Table 1). In a third experiment, OM73, an injection of FR involved portions of the basal, accessory basal, and lateral amygdaloid nuclei. This case had very many labeled axons in $\mathrm{Pa}$, with fewer labeled axons in $\mathrm{Pt}, \mathrm{Cdc}, \mathrm{Csl}, \mathrm{Cim}$, and $\operatorname{Re}$ (Table 1). Taken together, these experiments confirm that several parts of the amygdala have substantial projections to $\mathrm{Pa}$, with lighter input to other midline nuclei.

In three other experiments (OM43, OM44, and OM46), injections of BDA were made into medial hypothalamus, involving the region just rostral and/or caudal to the ventromedial hypothalamic nucleus (Table 1). In all of these, there is good anterograde axonal labeling in $\mathrm{Pa}$, with less labeling in $\mathrm{Pt}$ (Fig. 10B,C; Table 1). Labeled axons are also found in Cdc, CsI, Cim, and to a lesser degree in Re (Table 1).

\section{Pa innervation by 5-HT, ORX, and CRH}

Immunoreactive axons and axonal varicosities in Pa. In sections stained immunohistochemically for 5-HT, ORX, and $\mathrm{CRH}$, high concentrations of immunoreactive axons and varicosities, were found specifically in $\mathrm{Pa}$ (Fig. 11A-F, case OM74). In all cases the concentration of stained axons was much denser in $\mathrm{Pa}$ than in adjacent nuclei. The Pt was relatively free of stained axons.

Figure 5

Cases OM40, OM43, OM46. The distribution of labeled neurons in the thalamus following retrograde tracer injections into the striatum. Case numbers, tracers and injection sites are shown in the left column $(\mathrm{A}, \mathrm{F}, \mathrm{K}, \mathrm{P}, \mathrm{U})$, with the associated pattern of labeled neurons in the thalamus at four rostral-caudal levels to the right. Two animals (cases OM40 and OM43) each had injections of two different tracers. Dashed lines show approximate delineation of the accumbens core and shell based on our observations and previous reports (Meredith et al., 1996; Brauer et al., 2000). Tracer injection into the core and shell (A) resulted in labeled neurons in the dorsal midline nuclei, including paraventricu$\operatorname{lar}(\mathrm{Pa})$ and paratenial nuclei $(\mathrm{Pt})(\mathrm{B}-\mathrm{E})$. Tracer injection into the Sh (K) resulted in labeled neurons in $\mathrm{Pa}$, and to a lesser extent the $\mathrm{Pt}(\mathrm{L}-\mathrm{O})$ Injections into the putamen lateral to the accumbens nucleus $(F, P)$, resulted in labeled neurons ventral to $\mathrm{Pa}$ and $\mathrm{Pt}$, primarily in the central densocellular nucleus $(\mathbf{H}, \mathbf{I}, \mathbf{R})$, and in the central inferior nucleus. An injection into the medial caudate nucleus (U) resulted in labeled neurons in the central lateral superior nucleus $(X, Y)$, and some labeling in $\mathrm{Pa}$. No labeling in thalamus was found outside the areas shown. See also Table 1. Scale bars $=5 \mathrm{~mm}$ in A,F,K,P,U; $1 \mathrm{~mm}$ in B-E,G-J,L-O,Q-T,V-Y.
Double-labeling experiments. An injection of CTb-gold into the anterior dorsal midline thalamus involved $\mathrm{Pa}, \mathrm{Pt}$, and parts of the AM (Fig. 12A, case OM78). This injection was at approximately the same rostral-caudal level where high concentrations of $5-\mathrm{HT}, \mathrm{ORX}$, and $\mathrm{CRH}$ immunoreactive axons and axonal varicosities were found (Fig. 11, case OM74). The retrograde labeling was similar to that observed in case OM64, including labeled neurons in the perifornical area and paraventricular nucleus of the hypothalamus, the amygdala, and the dorsal and median raphe and other dorsal midbrain nuclei (Figs. 8, 9). In the dorsal and median raphe, there were concentrations of neurons labeled for 5-HT, intermingled with neurons retrogradely labeled with $\mathrm{CtB}$-gold. A few neurons double-labeled for both CtB-gold and 5-HT (Fig. 12B-D). In the perifornical area, there was dense immunohistochemical labeling for ORX. Again, a few neurons were double-labeled for both CtB-gold and ORX (Fig. 12E-G).

Neurons stained for either CtB-gold or CRH were found in close proximity to each other in the paraventricular hypothalamic nucleus (Fig. 13A), medial and basal amygdaloid nuclei (Fig. 13D), and lateral parabrachial nucleus and Barrington's nucleus ventrolateral to the PAG (Fig. 13E). Although the two types of stained neurons were intermingled, no doublelabeled neurons were found (Fig. 13A-E).

\section{DISCUSSION}

This study has shown in monkeys that $\mathrm{Pa}$, although very small in size, is connected to a remarkably extensive set of limbic, striatal, hypothalamic, and midbrain structures, similar to that in rats. These include connections with the accumbens nucleus, lateral septum, amygdala, entorhinal cortex, hippocampal formation, medial and lateral hypothalamus, dorsal raphe, PAG, and parabrachial nuclei. In addition, when the subcortical connections of $\mathrm{Pa}$ are considered in relation to its cortical connections that were detailed in a previous article (Hsu and Price, 2007), it is striking that the cortical areas related to $\mathrm{Pa}$ are themselves connected to many of the same subcortical structures as $\mathrm{Pa}$. That is, $\mathrm{Pa}$ is primarily connected to the "medial prefrontal network," which includes areas on the medial side of the frontal lobe, the medial edge of the orbital cortex, and a small region in the caudolateral orbital cortex (Öngür and Price, 2000); within this network, the strongest connections are with areas 25,32 , and $14 \mathrm{c}$, in the perigenual region (Hsu and Price, 2007). The medial prefrontal network, and particularly the peri-genual region, have strong and relatively distinct connections with the same parts of the striatum, amygdala, entorhinal cortex, hypothalamus, and PAG that are connected to Pa (An et al., 1998; Öngür et al., 1998; Ferry et al., 2000; Price, 2007). Altogether, the circuit related to $\mathrm{Pa}$ includes almost the entire limbic core of the brain, extending from the medial prefrontal cortex to the caudal midbrain.

$\mathrm{Pa}$ also contains a high density of axons and axonal varicosities immunoreactive for 5-HT and ORX, as well as a moderate density of axons and axonal varicosities immunoreactive for $\mathrm{CRH}$. Double labeling with a retrograde tracer injection into $\mathrm{Pa}$ and immunohistochemistry demonstrated that axons for ORX and 5-HT originate from the hypothalamus and the dorsal/median raphe, respectively. CRH neurons were intermingled with neurons that project to $\mathrm{Pa}$ in 


\section{A OM73}

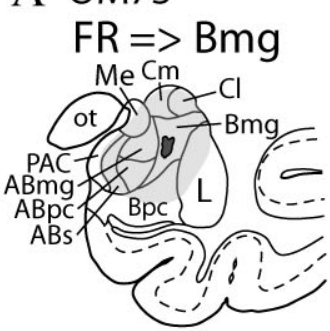

F OM78

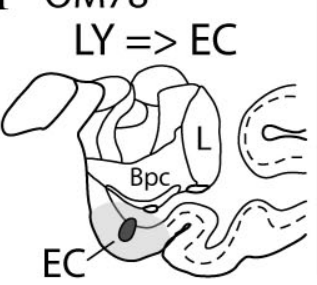

K OM16
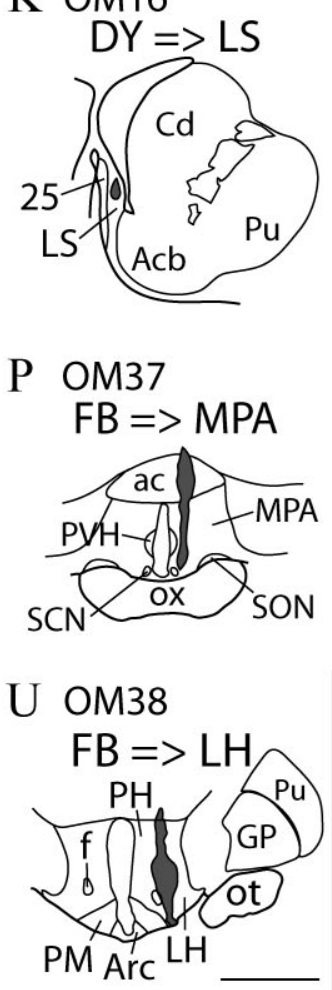
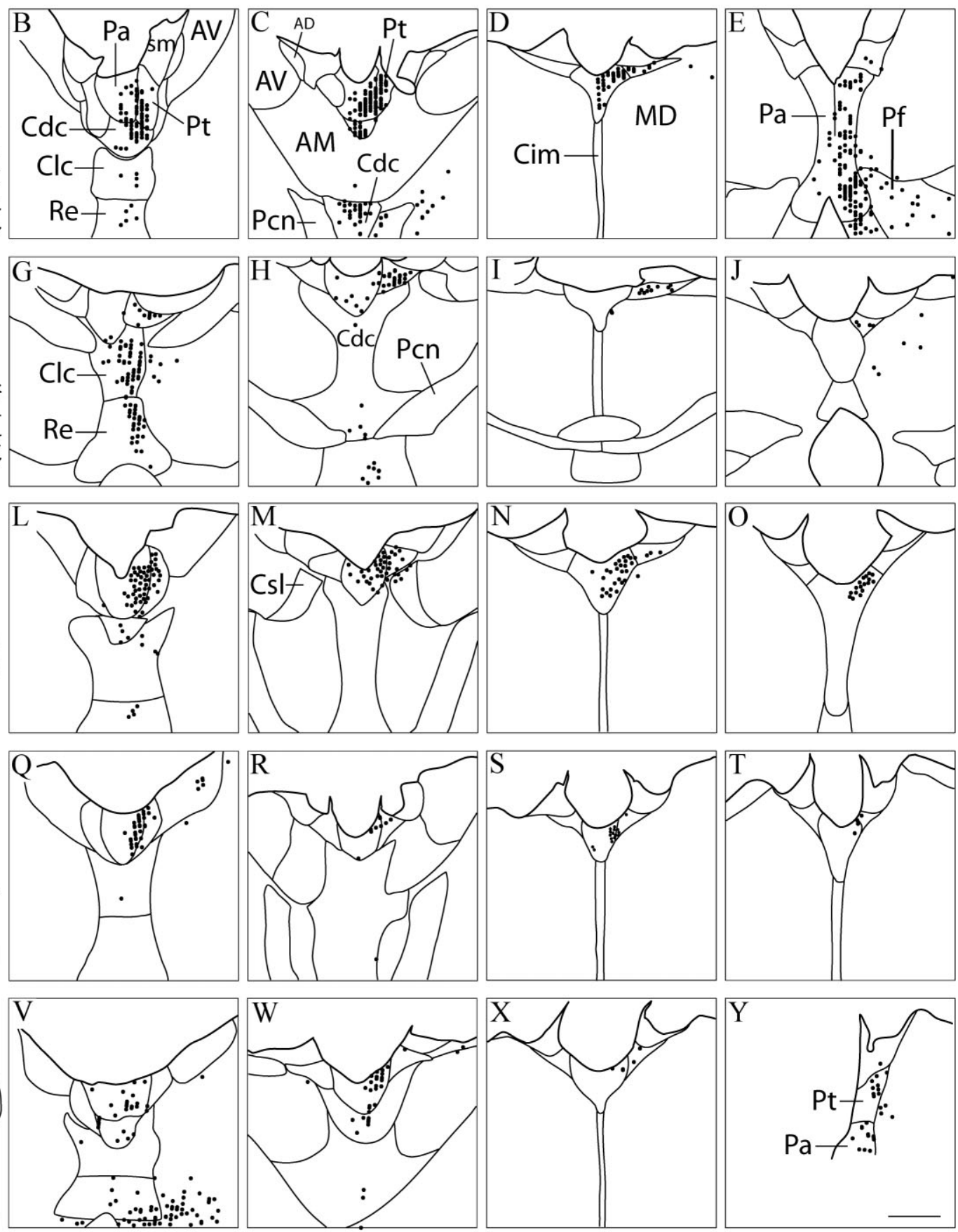

Figure 6.

Cases OM16, OM37, OM38, OM73, OM78. The distribution of labeled neurons in the thalamus following retrograde tracer injections into the magnocellular basal amygdaloid nucleus (A), entorhinal cortex (F), lateral septum (K), medial preoptic area (P), and lateral hypothalamus (U). Light gray shadings in $(A)$ and $(F)$ indicate spread of tracers fluro-ruby (FR) and lucifer yellow (LY). Injection sites were chosen according to Pattern of anterograde labeling following tracer injection into dorsal midline (see Figs. 1-3). Note that labeled neurons are concentrated in dorsal midline, primarily in Pa and Pt. No labeling in thalamus was found outside the areas shown. See also Table 1. Scale bars = $5 \mathrm{~mm}$ in A,F,K,P,U; $1 \mathrm{~mm}$ in B-E,G-J,L-O,Q-T,V-Y.

the amygdala and hypothalamus, although no double labeling was found.

\section{Pa output}

Accumbens nucleus. The accumbens nucleus, especially the shell region, had the heaviest innervation of any brain region labeled from an anterograde tracer injection into the dorsal midline thalamus. The retrograde tracer experiments also suggested that Pa projects primarily to the shell, while Pt may have more projection to the core region of the nucleus. This is consistent with a previous study of retrograde neuron labeling in $\mathrm{Pa}$ versus $\mathrm{Pt}$ following four retrograde tracer injections in the 


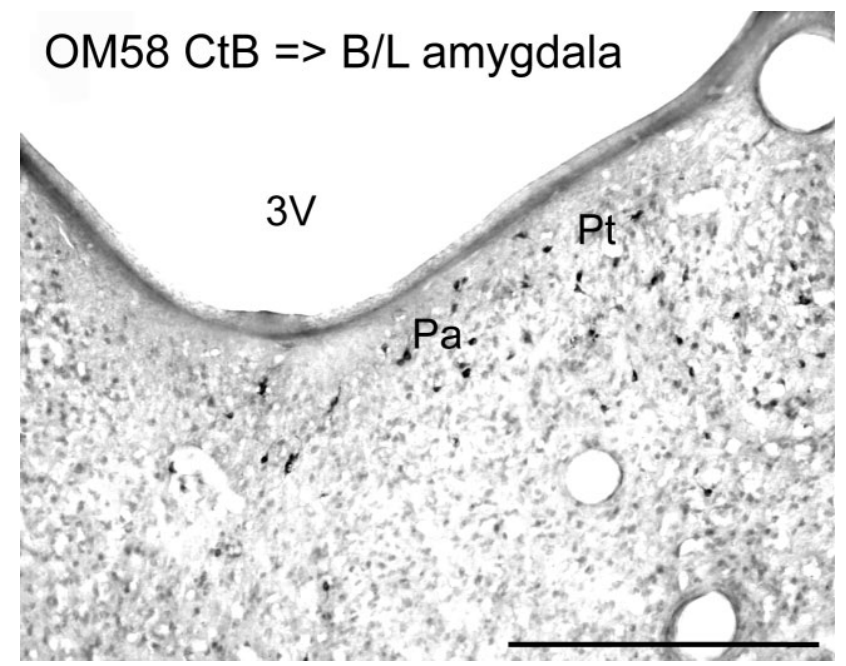

Figure 7.

Case OM58. Labeled neurons in the Pa and Pt following an injection of retrograde tracer CtB into the basal $(B)$ and lateral $(L)$ nuclei of the amgydala. See also Table 1. Scale bar $=500 \mu \mathrm{m}$.

ventral striatum of the macaque monkey (Giménez-Amaya et al., 1995). The same organization also exists in rats (Berendse and Groenewegen, 1990; Su and Bentivoglio, 1990; Freedman and Cassell, 1994; Vertes and Hoover, 2008). The shell of the accumbens is also specifically connected with cortical area 25 (Ferry et al., 2000), which is the area that is most strongly related to $\mathrm{Pa}$ (Hsu and Price, 2007). Beyond the accumbens nucleus, $\mathrm{Pa}$ and Pt project to the rostral-medial part of the caudate nucleus., which also receives a projection from the medial prefrontal network (Ferry et al., 2000).

Amygdala. The basal nucleus of the amygdala has strong reciprocal connections with $\mathrm{Pa}$ and $\mathrm{Pt}$, as well as with parts of Cdc. The present study showed very little projection from $\mathrm{Pa}$ to the central nucleus of the amygdala $(\mathrm{Ce})$, although this nucleus is a major target of $\mathrm{Pa}$ in rats ( $\mathrm{Su}$ and Bentivoglio, 1990; Moga et al., 1995; Li and Kirouac, 2008). The projection in rats arises primarily in the posterior part of $\mathrm{Pa}$, which was not injected in this study. Further, an anterograde tracer injection in $\mathrm{Ce}$ in the present study labeled a strong projection to posterior $\mathrm{Pa}$ (Fig. 10A). Previous studies in monkeys have shown that retrograde tracer injections into the amygdala label neurons in Pa (Aggleton et al., 1980; Mehler, 1980).

Entorhinal cortex. There are substantial interconnections between $\mathrm{Pa}$ and the entorhinal cortex, particularly its anterior part. Although a retrograde injection in the entorhinal cortex indicated that the projection originated primarily from the $\mathrm{Pt}, \mathrm{Clc}$, and $\mathrm{Re}$, with less from $\mathrm{Pa}$, this injection was just caudal to the major concentration of fibers from the thalamus. In contrast, a similar experiment in another study had retrogradely labeled neurons primarily in the anterior $\mathrm{Pa}, \mathrm{Clc}$, and $\mathrm{Re}$, with only occasional labeled neurons in the Pt (Insausti et al., 1987). It is likely that different subdivisions of the entorhinal cortex are connected to different midline thalamic nuclei. Studies in rats and cats also found that the entorhinal cortex receives projections from $\mathrm{Pa}$ (Yanagihara et al., 1987; Moga et al., 1995); electrical stimulation of the midline thalamus has further indicated a direct excitatory influence over both the amygdala and the entorhinal cortex (Zhang and Bertram, 2002).
Hypothalamus. There is a substantial projection from the dorsal midline thalamus to the hypothalamus, which appears from the retrograde tracer labeling to originate primarily in $\mathrm{Pa}$. In agreement with observations in rats, the projection is particularly dense rostrally in the medial preoptic area and suprachiasmatic nucleus (Moga et al., 1995), but there is also a substantial projection to more caudal parts of both medial and lateral hypothalamus. Only a few axons from $\mathrm{Pa}$ were found in the cellular part of the paraventricular hypothalamic nucleus, but there were many axons immediately lateral to this nucleus. Light and electron microscope studies in rats have shown that dendrites of the magnocellular neurons in the paraventricular hypothalamic nucleus extend laterally out into this region (Silverman and Oldfield, 1984; Oldfield and Silverman, 1985). It is likely that the axons from $\mathrm{Pa}$ synapse on these dendrites. In contrast to findings in rats (Moga et al., 1995), relatively little projection was found the central part of the ventromedial hypothalamic nucleus.

Lateral septum. The lateral septum had moderate levels of labeling following an anterograde tracer injection into the dorsal midline. A small retrograde tracer injection into the lateral septum showed that this projection came from $\mathrm{Pa}$, and to a lesser extent, the Pt. This pattern of labeling is consistent with previous studies in rats (Berendse and Groenewegen, 1990; Moga et al., 1995).

\section{Inputs to $\mathbf{P a}$}

Hypothalamus. Retrogradely labeled neurons from $\mathrm{Pa}$ were found in several hypothalamic nuclei including the suprachiasmatic nucleus, medial preoptic nucleus, ventromedial nucleus, arcuate nucleus, and perifornical area. The suprachiasmatic nucleus is well known to project densely to $\mathrm{Pa}$ in rats (Watts and Swanson, 1987; Watts et al., 1987). In the study by Watts and Swanson (1987), retrograde labeling from $\mathrm{Pa}$ was found mostly in the suprachiasmatic nucleus, and in an area of the medial preoptic nucleus just dorsal to it. In the present study, retrograde labeling had a broader dorsal-ventral distribution in the medial preoptic nucleus. No rat studies have examined hypothalamic inputs to $\mathrm{Pa}$ posterior to the suprachiasmatic nucleus, but the present study found additional inputs from the ventromedial nucleus, arcuate nucleus, and perifornical area.

Many of the neurons in the perifornical area are immunoreactive for ORX (hypocretin) (Horvath et al., 1999; present results). Consistent with rat studies (Peyron et al., 1998; Nambu et al., 1999; Kirouac et al., 2005), this study demonstrated that the primate $\mathrm{Pa}$ contained the highest density of ORX-positive axons and axonal varicosities in the thalamus. As indicated by the double-labeling experiments, at least some of the ORX neurons in the perifornical area of the hypothalamus project to $\mathrm{Pa}$, consistent with the finding in rats (Kirouac et al., 2005). The non-ORX neurons in the ventromedial hypothalamus and arcuate nucleus may contain other hypothalamic peptides including neuropeptide $\mathrm{Y}$, substance $\mathrm{P}$, somatostatin, neurotensin, and endomorphin 2 (Langevin and Emson, 1982; Gray and Morley, 1986; Larsen, 1992; Pierce and Wessendorf, 2000), all of which densely innervate $\mathrm{Pa}$ (Freedman and Cassell, 1994; Pierce and Wessendorf, 2000; Uroz et al., 2004; Otake, 2005).

The present study also found moderate levels of $\mathrm{CRH}$ axons and axonal varicosities in $\mathrm{Pa}$, consistent with previous findings in rats and monkey (Swanson et al., 1983; Foote and Cha, 

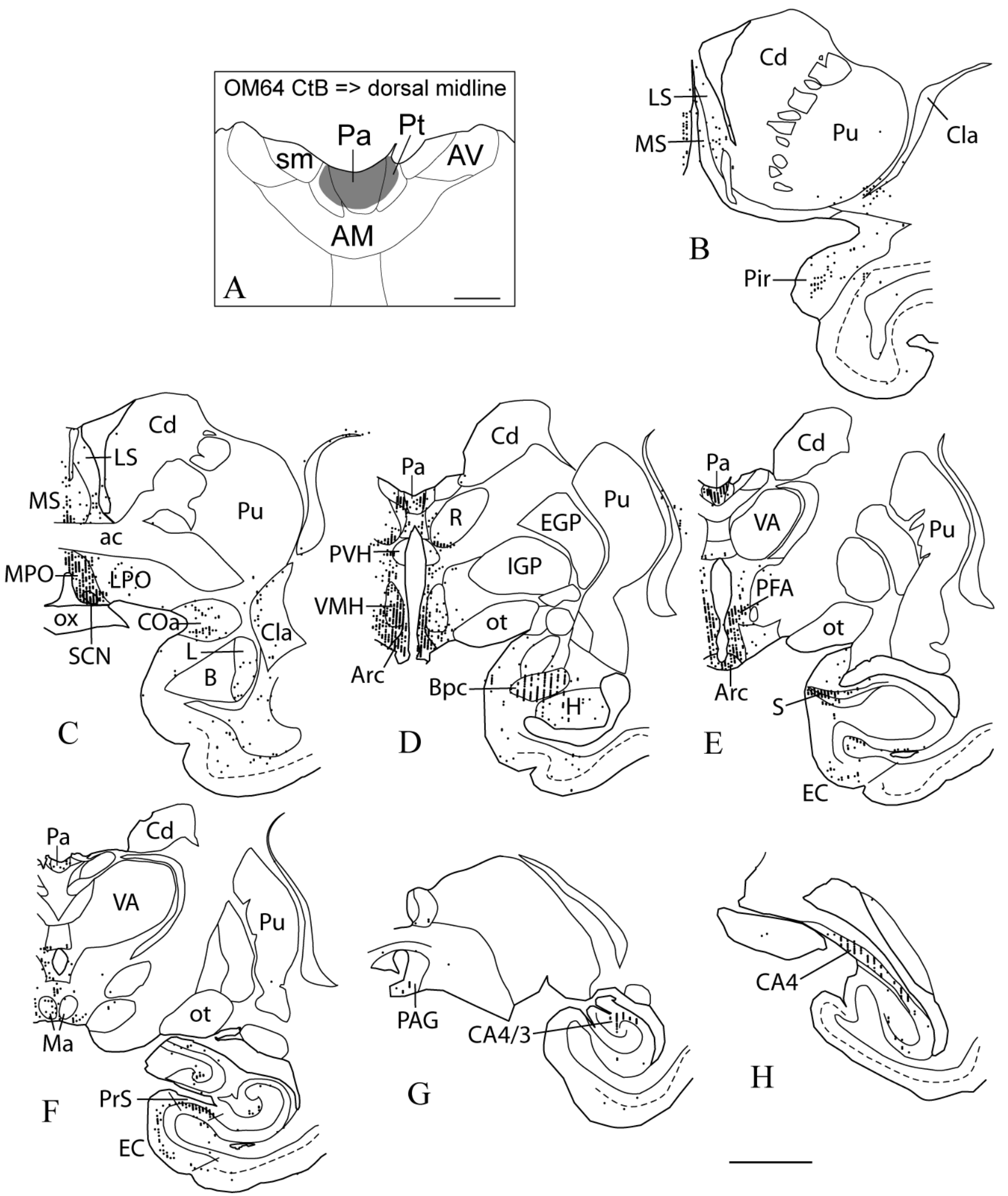

Figure 8.

Case OM64. Retrograde tracer CtB injection into the dorsal midline thalamus (A), and the resulting labeling in several subcortical structures (rostral-caudal, B-H). Each dot represents one labeled neuron. The dashed lines show layer IV or the border between layers III and V in the temporal cortex. High numbers of labeled neurons were found in the hypothalamus, particularly in the suprachiasmatic nucleus, medial preoptic nucleus, ventromedial hypothalamus, arcuate nucleus, and perifornical area (C-E). High numbers were also found in the subiculum (E) and presubiculum $(\mathrm{F})$. Moderate levels were found in the anterior cortical nucleus of the amygdala $(C)$, basal nucleus of the amygdala, parvicellular division (D), entorhinal cortex $(F)$, and hippocampus (CA4/3, CA4) $(G, H)$. Scale bars $=1 \mathrm{~mm}$ in $A ; 5 \mathrm{~mm}$ in $B-H$. 


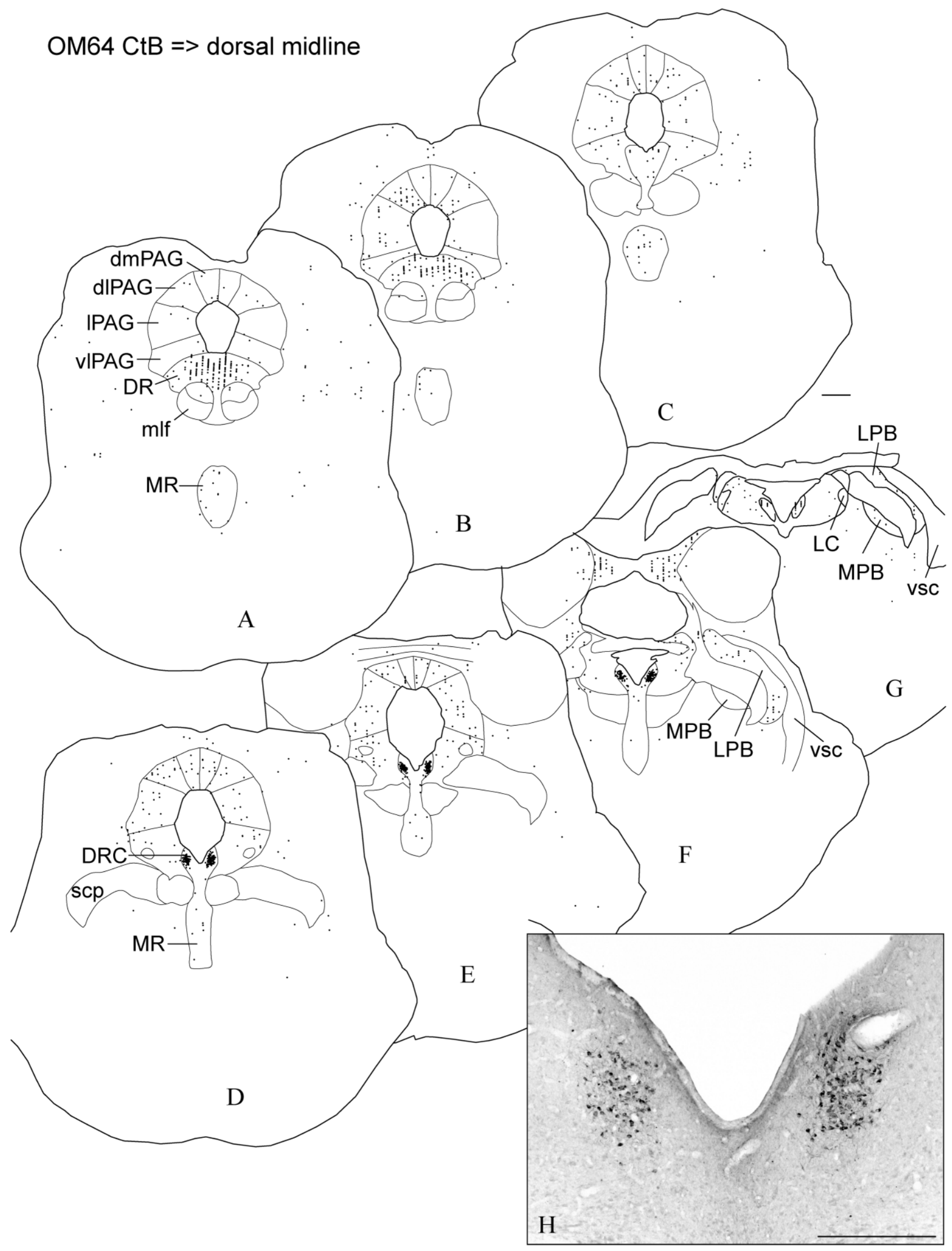

Figure 9.

Case OM64. The distribution of labeled neurons in the midbrain (rostral-caudal, A-G) following an injection of the retrograde tracer CtB into the dorsal midline thalamus. H: Photomicrograph of labeled cells in the dorsal raphe. Note the high density of labeled neurons in the caudal division of the dorsal raphe. Labeled neurons were also found in the PAG and the lateral and medial parabrachial nucleus. Scale bars = $1 \mathrm{~mm}$ in A-G; $500 \mu \mathrm{m}$ in $\mathrm{H}$. 
1988; Otake and Nakamura, 1995). CRH neurons were located primarily in the paraventricular hypothalamic nucleus, in the lateral parabrachial nucleus and Barrington's nucleus in the midbrain, and to a lesser degree in the amygdala. A doublelabeling study in rats found that $\mathrm{CRH}$ innervation of $\mathrm{Pa}$ originates from all of these sites (Otake and Nakamura, 1995). In this study, many retrogradely labeled neurons from an injection of CtB-gold in $\mathrm{Pa}$ were found in close proximity to the $\mathrm{CRH}$ neurons in all these sites, but no double labeling was found. This may be because the CtB injection was restricted to the anterior part of $\mathrm{Pa}$; in addition, detection of $\mathrm{CRH}$ in rats is often enhanced by the use of colchicine (e.g., Swanson et al., 1983), which was not used in this study.

Midbrain. As expected, projections to Pa were found from many neurons in the dorsal raphe, as well as more moderate projections from the median raphe, PAG, and parabrachial nucleus. This is consistent with findings in rats, where major brainstem inputs to $\mathrm{Pa}$ originate from these regions in addition to the locus coeruleus, nucleus of the solitary tract, and ventral medulla (Krout and Loewy, 2000a,b; Otake et al., 2002). The present study found a projection from the lateral portion of parabrachial nucleus to the anterior $\mathrm{Pa}$ (Fig. 7). This topography is consistent with findings in rats, in which the external lateral parabrachial subnucleus projects strongly to the anterior $\mathrm{Pa}$, and less so to the middle and posterior Pa (Krout and Loewy, 2000a).

Vertes (1991) injected the anterograde tracer PHA-L into the dorsal raphe in rats and found labeled axons in the posterior $\mathrm{Pa}$. In the present study retrograde tracer injected into the anterior $\mathrm{Pa}$ showed that the majority of these projections originated from the caudal division of the dorsal raphe. Consistent with the present study, Vertes et al. (1999) also found that the median raphe projects to $\mathrm{Pa}$.

In addition to its high concentration of ORX, Pa contains the highest concentration of 5-HT-immunoreactive axons and axonal varicosities in the primate thalamus (Lavoie and Parent, 1991; this study, Fig. 8A). By combining retrograde tracing with immunoreactivity for 5-HT, it was also demonstrated that 5-HT innervation of $\mathrm{Pa}$ originates from both the dorsal and median raphe nuclei (Fig. 9). In this experiment (case OM78), the number of double-labeled neurons were too few to determine rostral-caudal topography, however the section shown in Figure 9 is from the mid-caudal level, and contained the highest number of double-labeled neurons.

Amygdala. Input to the anterior part of $\mathrm{Pa}$ from the amygdala originates primarily in the parvocellular part of the basal amygdaloid nucleus. In contrast, the posterior part of $\mathrm{Pa}$ receives fibers from Ce (Price and Amaral, 1981; this study). Consistent with this, amygdala projections to $\mathrm{Pa}$ have been shown in rats (Cornwall and Phillipson, 1988; Chen and Su, 1990).

Subiculum, presubiculum, entorhinal cortex, and hippocampus. In the present study, retrograde tracer injection into $\mathrm{Pa}$ resulted in moderate to dense retrograde labeling in

Figure 10.

Cases DM28, OM44, OM46. The distribution of anterograde labeling in the $\mathrm{Pa}$ and $\mathrm{Pt}$ following injections of tritiated leucine $(3 \mathrm{H})$ in the central nucleus of the amygdala $(\mathrm{Ce})$ and intra-amygdaloid part of the bed nucleus of the stria terminalis (A), BDA in the medial hypothalamus (B), and BDA in the ventromedial hypothalamus (C). Following the anterograde injection in Ce/BSTIA, labeling is concentrated in the posterior part of $\mathrm{Pa}(\mathrm{A})$. See also Table 1 . Scale bars $=1 \mathrm{~mm}$ in $\mathrm{A}, \mathrm{C} ; 500 \mu \mathrm{m}$ in B.
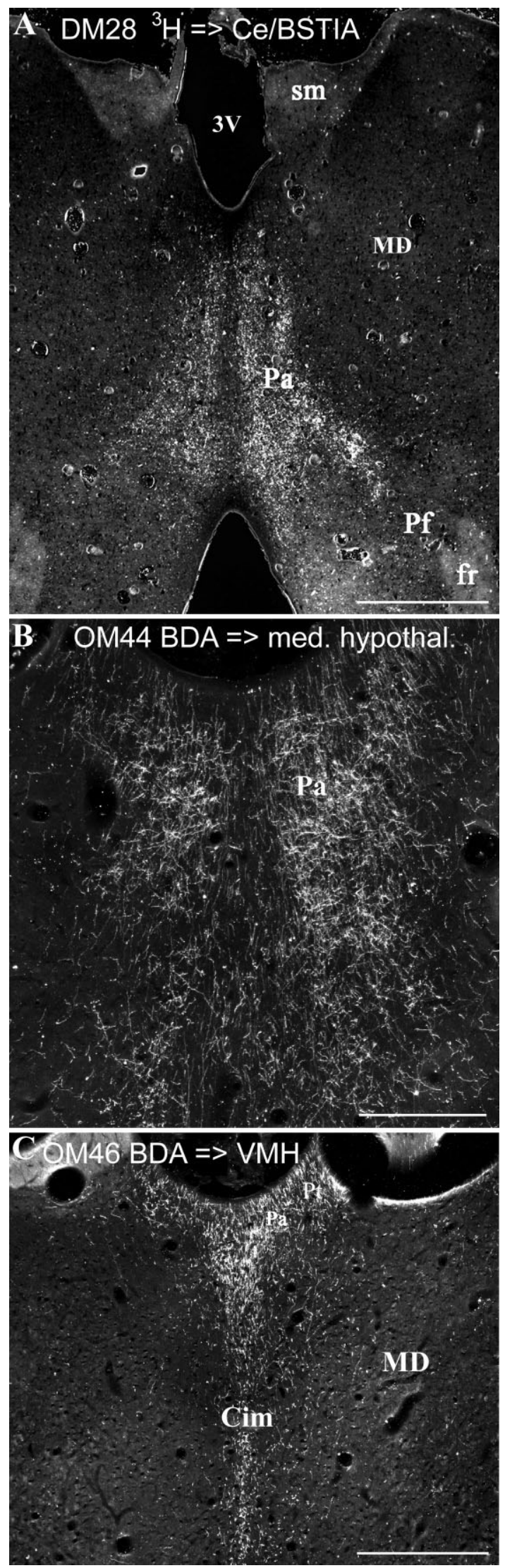

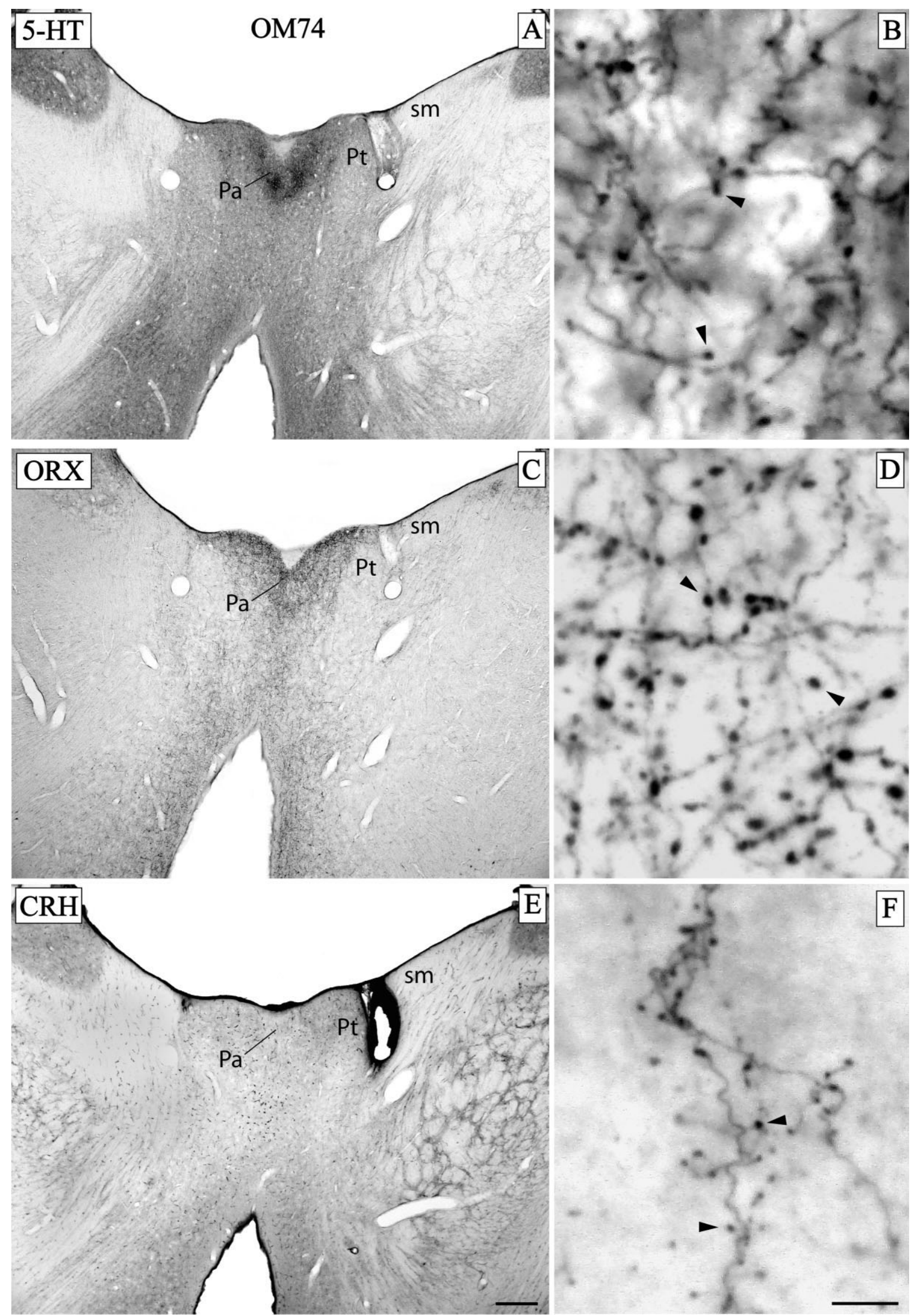

Figure 11.

Case OM74. Adjacent sections from the same animal, through the anterior part of the thalamus stained for serotonin (5-HT) (A), orexin (ORX) (C), and corticotropin-releasing hormone (CRH) (E). High densities of axons and axonal varicosities staining for 5-HT and ORX were found in Pa $(A, C)$, and moderate densities were found for CRH (E). In the right column, high power photomicrographs taken from an area within Pa show high densities of labeled axons and axonal varicosities (arrowheads) staining for $5-\mathrm{HT}$ (B), ORX (D), and lesser for CRH (F). Scale bars $=500$ $\mu \mathrm{m}$ in $\mathrm{A}, \mathrm{C}, \mathrm{E} ; 10 \mu \mathrm{m}$ in B,D,F. 

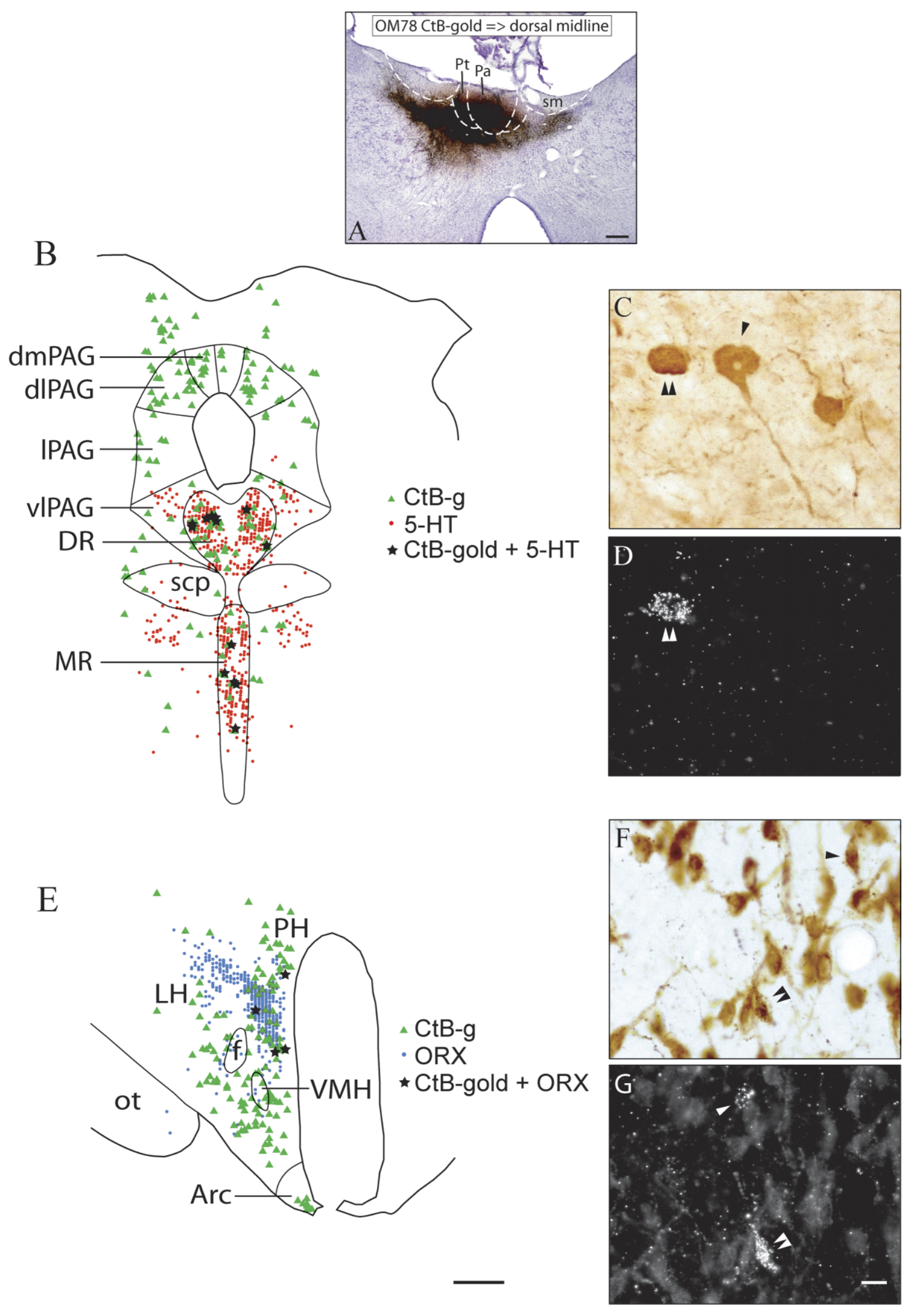

Figure 12 
the subiculum, presubiculum, entorhinal cortex, and CA4/3 subfields of the hippocampus. Previous studies have found that the projections from these structures terminate predominantly in the reuniens nucleus, anterior ventral, and lateral dorsal nuclei (Aggleton et al., 1986; McKenna and Vertes, 2004; Saunders et al., 2005). However, the subiculum and entorhinal cortex were found to project to $\mathrm{Pa}$ as well (Aggleton et al., 1986; Saunders et al., 2005), and the entorhinal projection to $\mathrm{Pa}$ appears to be unique to primates. Although previous studies have not reported hippocampal or presubiculum projections to $\mathrm{Pa}$, this may be because projections to $\mathrm{Pa}$ shown in the present study originated from the presubiculum and caudal CA4/3, which do not appear to have been targeted with anterograde tracers.

\section{Functional considerations}

$\mathrm{Pa}$ has been proposed to have a role in arousal, stress, energy balance, salt appetite, and circadian rhythms (Van der Werf et al., 2002; Kelley et al., 2005; Shekhtman et al., 2007). These functions have been suggested for Pa primarily because of its connections with related midbrain and hypothalamic structures, and its remarkably dense innervation by axons containing monoamines and neuropeptides such as CRH and ORX (Otake and Nakamura, 1995; Van der Werf et al., 2002; Kirouac et al., 2005; this study). Studies have shown changes in levels of the molecular marker c-fos in Pa following acute stress (e.g., Senba et al., 1993; Beck and Fibiger, 1995; Cullinan et al., 1996), in anticipation of feeding (Nakahara et al., 2004; Angeles-Castellanos et al., 2007), and during the circadian rhythm (Novak and Nunez, 1998); lesions of $\mathrm{Pa}$ also indicate its role in these processes (e.g., Salazar-Juárez et al., 2002; Nakahara et al., 2004; Landry et al., 2007).

A remarkable feature of $\mathrm{Pa}$ is that it is consistently and strongly activated following a wide variety of stressors including conditioned fear, restraint, handling, swim, mild footshock, air puff, sleep deprivation, salt deprivation, hypoxia, and hypoglycemia (Chastrette et al., 1991; Sharp et al., 1991; Duncan et al., 1993; Imaki et al., 1993; Senba et al., 1993; Beck and Fibiger, 1995; Cullinan et al., 1996; Bhatnagar and Dallman, 1998; Bubser and Deutch, 1999; Sica et al., 2000; Semba et al., 2001; Otake et al., 2002; Spencer et al., 2004; Arbelaez et al., 2007). Presumably, information about these forms of stress reaches $\mathrm{Pa}$ through sources such as the PAG, parabrachial nucleus, raphe, and hypothalamus. The large number and variety of these forms of stress suggests that $\mathrm{Pa}$ plays a role in stress in general, and is

\section{Figure 12.}

Case OM78. Injection of CtB-gold into the anterior part of the dorsal midline thalamus (A), followed by staining for 5-HT (B-D) or ORX (E-G). Double-labeled neurons for CtB-gold and 5-HT were found in the dorsal and median raphe (B). High-power photomicrographs of the same field show neurons staining positively for 5-HT in brightfield (C), and CtB-gold in darkfield (D). A single arrowhead indicates a neuron staining positively for 5-HT (C); a double arrowhead indicates a neuron staining for both 5-HT and CtB-gold (C,D). Double-labeled neurons for CtB-gold and ORX were found in the perifornical area of the medial hypothalamus (E). High-power photomicrographs of the same field show neurons staining positively for ORX in brightfield (F), and CtBgold in darkfield (G). Single arrowheads indicate neurons staining positively for ORX (F) or CtB-gold (G). A double arrowhead indicates a neuron staining for both ORX and CtB-gold $(F, G)$. The photomicrograph (C) was constructed using different focal points for the three neurons. Scale bars $=500 \mu \mathrm{m}$ in $A ; 1 \mathrm{~mm}$ in B,E; $10 \mu \mathrm{m}$ in C,D,F,G. not specific for any given type. Indeed, the relatively small size of $\mathrm{Pa}$ makes it questionable whether it would have the necessary neural machinery to distinguish and react to different types or causes of stress in detail. Instead, it may be suggested that $\mathrm{Pa}$ plays a relatively specific role, but one that is common to all forms of stress.

Perhaps the most convincing suggestion is that $\mathrm{Pa}$ plays a unique role in regulating the adaptation to chronic stress. Rats with a history of repeated stress exhibit habituation of the hypothalamic-pituitary-adrenal (HPA) axis if a subsequent stressor is the same type as the repeated stressor; in contrast, the response of the HPA axis is enhanced if the subsequent stressor is different from the repeated stressor. Both of these effects are dependent on an intact $\mathrm{Pa}$, particularly its posterior division (Bhatnagar and Dallman, 1998; Bhatnagar et al., 2002, 2003), and may be related to corticosterone-mediated negative feedback onto Pa (Jaferi et al., 2003). In these studies, lesions of the posterior $\mathrm{Pa}$ have no effect on the HPA response to an acute stressor, suggesting that $\mathrm{Pa}$ has a unique role in regulating chronic stress.

A recent study of neural activity related to the autonomic response to hypoglycemia suggests that the same mechanism is present in humans in relation to a different cause of stress. Hypoglycemia produces a stress-like response that includes both autonomic and endocrine components, which help to overcome the effects of the inadequate blood sugar. If the hypoglycemia is continued, however, the response habituates, constituting an important problem with diabetics who mistakenly administer excess insulin. With positron emission tomography (PET), it was found that on the first day of hypoglycemia in normal subjects there is increased synaptic activity in a network of interconnected brain regions including medial and orbital prefrontal cortex, thalamus, and PAG (Teves et al., 2004). On the second day of chronic hypoglycemia, however, there was a specific increase in synaptic activity in the dorsal midline thalamus that was associated with the attenuation of the stress response (Arbelaez et al., 2007). Like the rat experiments with restraint stress, this suggests that $\mathrm{Pa}$ and other dorsal midline thalamic nuclei play an active inhibitory role in reducing responses to chronic hypoglycemia.

$\mathrm{Pa}$ connections and neurochemical input, combined with its role in regulating chronic stress, also suggest that it may play a role in stress-related psychopathology. The connections of $\mathrm{Pa}$ shown in this and previous studies in rats and monkeys (Moga et al., 1995; Bubser and Deutch, 1999; Krout and Loewy, 2000a,b; Hsu and Price, 2007; Vertes et al., 2008), suggest that $\mathrm{Pa}$ receives stress-related signals from the hypothalamus, dorsal raphe, parabrachial nuclei, and PAG, and then has an influence over structures such as the amygdala, accumbens nucleus, and the subgenual cortex (Fig. 14). Interestingly, all of these structures are involved in mood disorders, substance abuse disorders, and related clinical conditions whose etiology may include a history of stress or abnormal reactions to stress (Drevets et al., 1997; Drevets, 1999; Breiter and Rosen, 1999; Epstein et al., 2006). A previous study in primates showed strong connections between $\mathrm{Pa}$ and the subgenual cortex (Hsu and Price, 2007), which exhibits abnormal activity in major depressive disorder (e.g., Drevets et al., 1997; Mayberg et al., 1999; Pizzagalli et al., 2004; Périco et al., 2005; Siegle et al., 2006; Van Laere et al., 2006). A functional MRI (fMRI) study has also found that the subgenual cortex is abnor- 
A

OM78 CtB-gold => dorsal midline
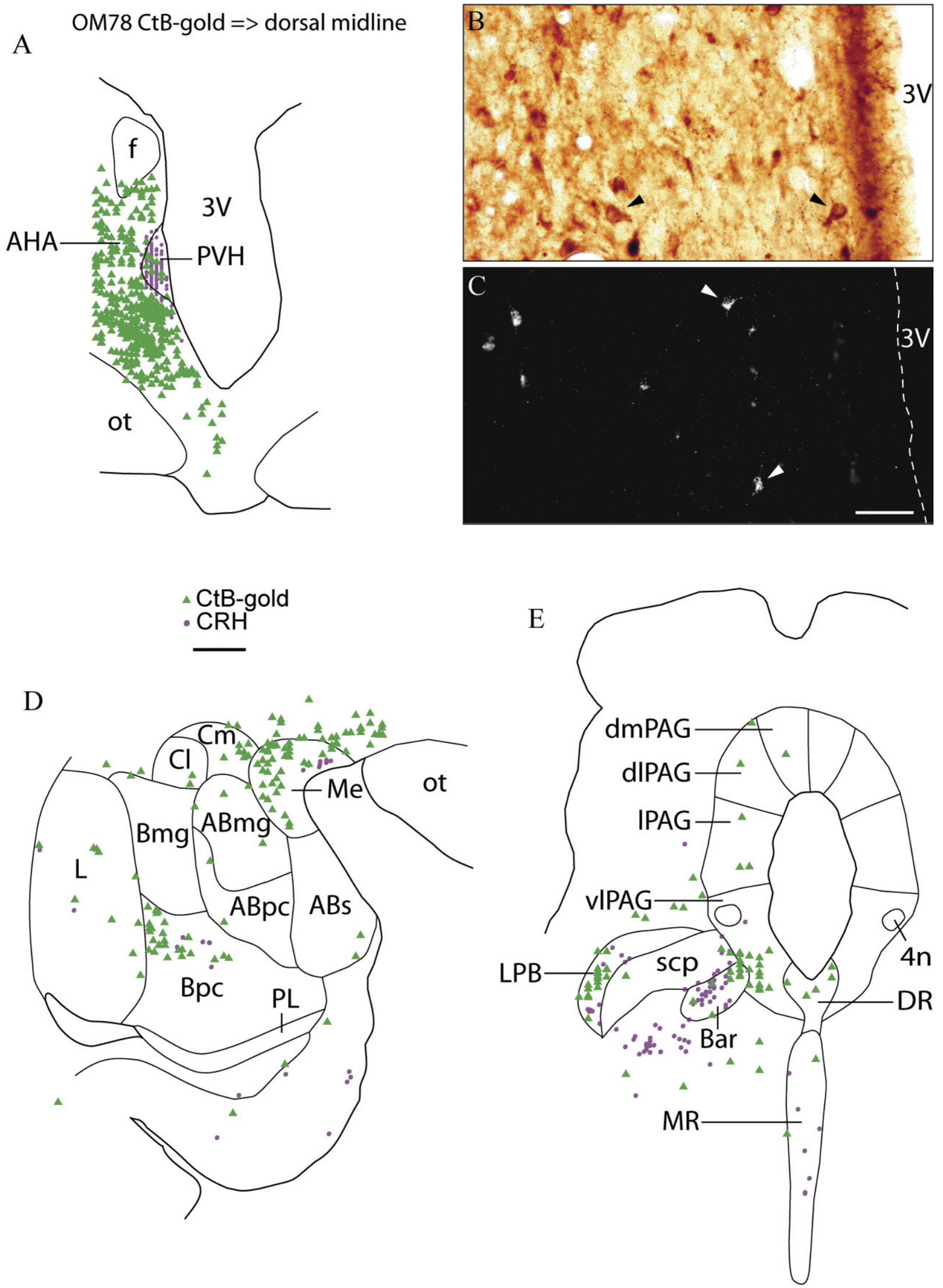

$\mathrm{D}$

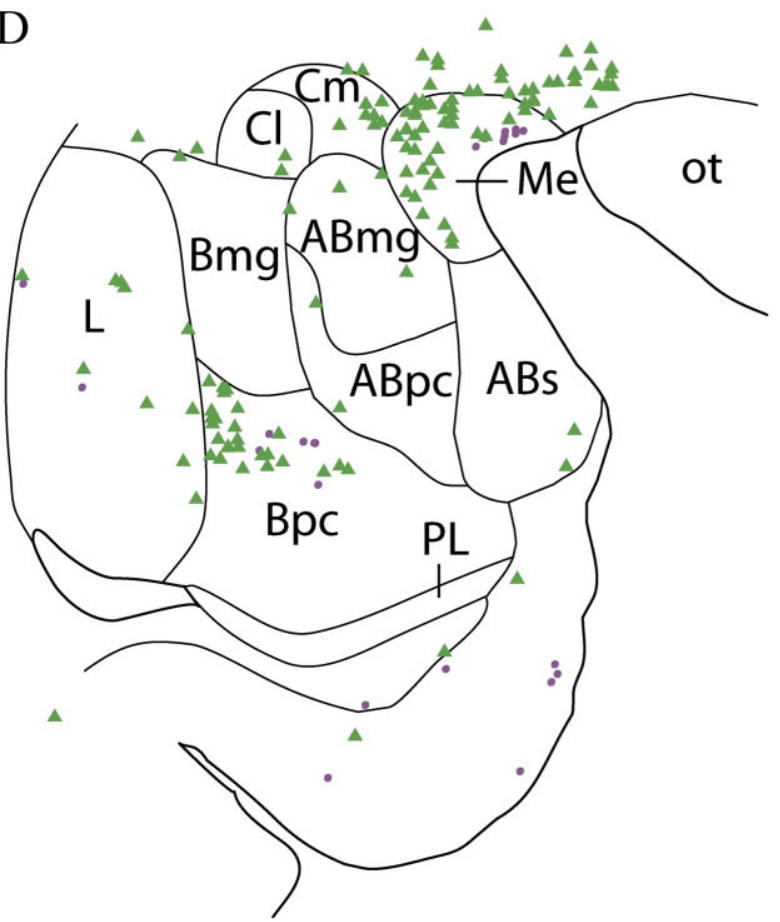

Figure 13.

Case OM78. Injection of CtB-gold into the anterior part of the dorsal midline thalamus followed by staining for CRH (same case as in Fig. 9). Although neurons staining for CtB-gold and $\mathrm{CRH}$ were found in similar locations, no double-labeled neurons were found. This included the paraventricular hypothalamic nucleus (A), medial amygdala and parvicellular division of the basal nucleus of the amygdala (D), lateral parabrachial, and Barrington's nucleus (E). High-power photomicrographs of the same field show neurons staining positively for CRH in brightfield (B, single arrowheads), and CtB-gold in darkfield (C, single arrowheads). Scale bars $=1 \mathrm{~mm}$ in A,D,E; $50 \mu \mathrm{m}$ in B,C. 


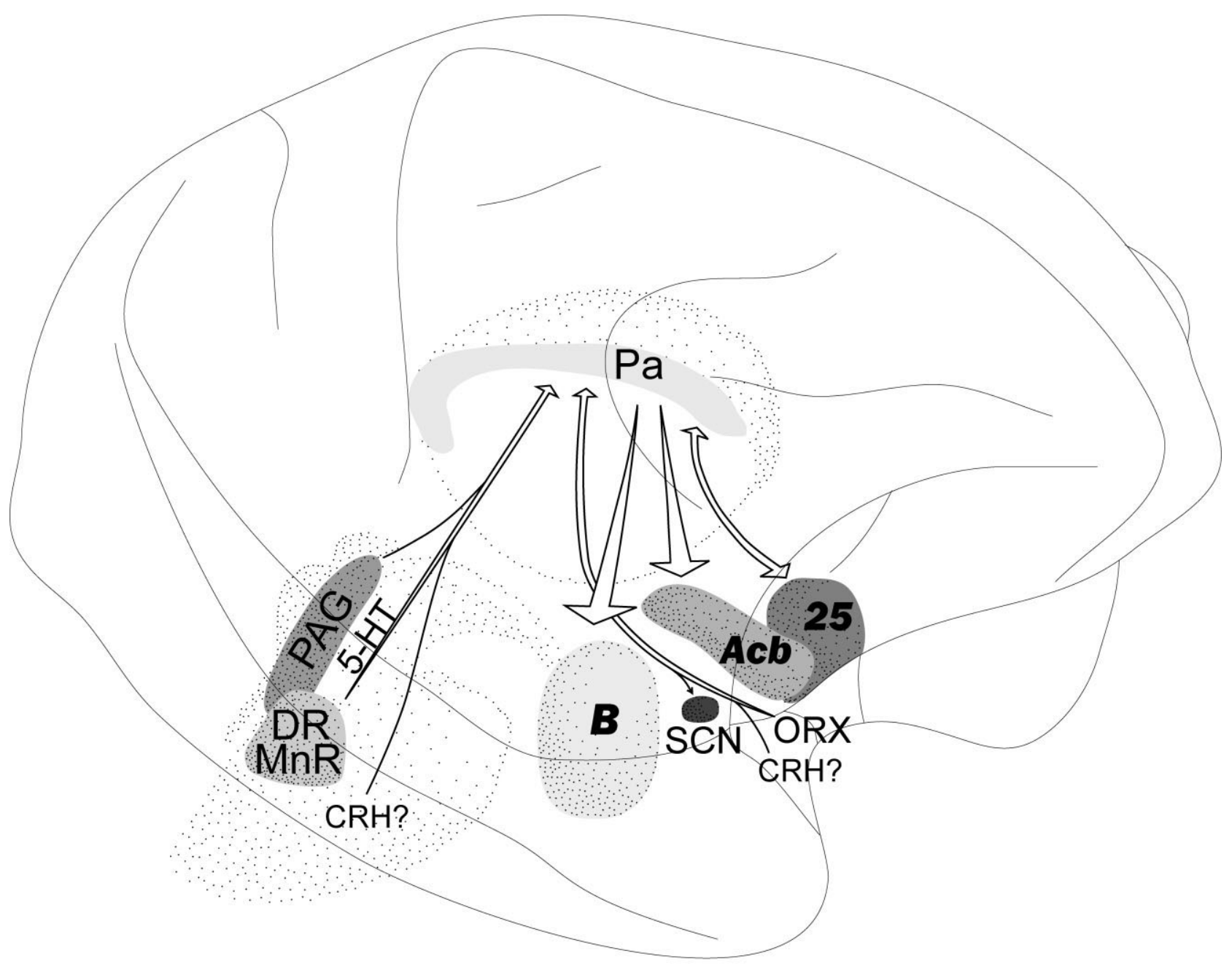

Figure 14.

Major connections of the primate Pa suggest that it may be a nodal area within a circuit that includes the PAG, dorsal raphe, median raphe, hypothalamus, amygdala, accumbens nucleus, and subgenual prefrontal cortex (area 24/25). The Pa has been especially linked to the stress response, but the circuit may also be involved in major depressive disorder and substance abuse. In this study 5-HT innervation of Pa was shown to originate from dorsal and median raphe, and ORX innervation from the hypothalamus; CRH innervation may originate from the midbrain and/or hypothalamus (see Discussion).

mally coupled with the midline thalamus in depressed subjects compared to controls (Greicius et al., 2007).

In addition to the wide circuit that $\mathrm{Pa}$ is part of, it is remarkable that the nucleus receives very strong inputs from several systems that are defined by their specific neurotransmitters. In this report we have focused on 5-HT, CRH, and ORX, and $\mathrm{Pa}$ also receives a dense input of norepinephrinergic and dopaminergic fibers (Sánchez-González et al., 2005; Vogt et al., 2008). The full significance of these inputs is difficult to discern at this time, but it may be presumed that they contribute importantly to the role of $\mathrm{Pa}$ in responses to stress and in stress-related disorders.

The majority of the serotonergic projections to $\mathrm{Pa}$ originate from the caudal division of the dorsal raphe. Studies in rats show that the caudal DR projects strongly to limbic structures including the amygdala and hippocampus, while the rostral DR projects more generally to the striatum, sub- stantia nigra, and neocortex (Imai et al., 1986; Commons et al., 2003; Abrams et al., 2004), suggesting that the caudal DR is selectively involved in mediating emotional behavior. The middle and caudal DR are selectively activated following inescapable vs. escapable shock, as shown by c-fos (Grahn et al., 1999), and injections of CRH into the caudal but not rostral DR mimicked the behavior following inescapable shock or exposure to social defeat (Hammack et al., 2002; Gardner et al., 2005). CRH-induced activation of DR neurons is also stronger in caudal compared to rostral DR (Meloni et al., 2008). Taken together, these observations suggest a specific role for the caudal DR in mediating emotional behavior.

$\mathrm{Pa}$ receives a dense innervation from hypothalamic ORX neurons (Peyron et al., 1998; this study), as part of a larger projection of the ORX neurons that includes the subgenual medial prefrontal cortex (which itself is strongly related to $\mathrm{Pa}$; 
Hsu and Price, 2007) as well as the norepinephrinergic, serotonergic, and CRH cell groups (Baldo et al., 2003; Saper et al., 2005). The action of the ORX fibers in all these sites appears to be depolarizing and excitatory (Ishibashi et al., 2005). This action on the norepinephrinergic and serotonergic is part of the role of ORX to promote wakefulness and to stabilize the wake/sleep cycle; the absence of ORX in this system results in narcolepsy in which the wake/sleep cycle becomes unstable such that episodes of sleep suddenly intrude onto wakefulness (Sutcliffe and de Lecea, 2000; Harris et al., 2005; Saper et al., 2005). Given the proposed function of the $\mathrm{Pa}$ in suppressing the chronic response to stress (see above), it is tempting to suggest that the interaction of ORX with the Pa may play a role in stress similar to that in sleep. That is, ORX might promote and stabilize the normal response to stress in acute situations, but then allow the response to be effectively and consistently suppressed when the stress becomes chronic.

ORX has also been shown to play a role in reward-seeking behavior, drug relapse and addiction (Harris et al., 2005), and in mediating stress-induced reinstatement of drug-seeking behavior (Boutrel et al., 2005). It is striking that in addition to ORX connections to $\mathrm{Pa}$ and the subgenual prefrontal cortex (above) there are many ORX fibers to the shell of the accumbens nucleus, which is also strongly connected to both $\mathrm{Pa}$ and the subgenual cortex (Ferry et al., 2000; Baldo et al., 2003; this study). The accumbens nucleus is known for its role in substance abuse disorders (Deadwyler et al., 2004) and may also play a role in anhedonia in major depression (e.g., Schlaepfer et al., 2008). Thus, this circuit may be involved in both disorders, which are often preceded by chronic stress and are highly comorbid (Brady and Sinha, 2005).

In summary, the circuitry of the primate $\mathrm{Pa}$ and its neurochemical inputs, shown in this (Fig. 14) and other studies, suggest that Pa may be a nodal point that could function to limit responses to chronic stress. Dysfunction within this circuit, leading to continued and excessive response to chronic stress, may underlie at least part of the mechanism by which stress triggers or promotes disorders in mood and substance abuse.

\section{ACKNOWLEDGMENTS}

We thank Mr. Hieu Van Luu, Mrs. Van Nguyen, and Ms. Cristina Mazuski for excellent technical assistance. The content is solely the responsibility of the authors and does not necessarily represent the official views of the National Institute of Mental Health or the National Institutes of Health.

\section{LITERATURE CITED}

Abrams JK, Johnson PL, Hollis JH, Lowry CA. 2004. Anatomic and functional topography of the dorsal raphe nucleus. Ann N Y Acad Sci 1018:46-57.

Aggleton JP, Burton MJ, Passingham RE. 1980. Cortical and subcortica afferents to the amygdala of the rhesus monkey (Macaca mulatta). Brain Res 190:347-368.

Aggleton JP, Desimone R, Mishkin M. 1986. The origin, course, and termination of the hippocampothalamic projections in the macaque. 243:409-421.

Amaral DG, Price JL. 1984. Amygdalo-cortical projections in the monkey (Macaca fascicularis). J Comp Neurol 230:465-496.

An X, Bandler R, Öngür D, Price JL. 1998. Prefrontal cortical projections to longitudinal columns in the midbrain periaqueductal gray in macaque monkeys. J Comp Neurol 401:455-479.

Angeles-Castellanos M, Mendoza J, Escobar C. 2007. Restricted feeding sched- ules phase shift daily rhythms of c-Fos and protein Per1 immunoreactivity in corticolimbic regions in rats. Neuroscience 144:344-355.

Arango V, Underwood MD, Mann JJ. 2002. Serotonin brain circuits involved in major depression and suicide. Prog Brain Res 136:443-453.

Arbelaez AM, Powers WJ, Videen TO, Price JL, Cryer PE. 2007. Attenuation of counterregulatory responses to recurrent hypoglycemia by active thalamic inhibition: a mechanism for hypoglycemia-associated autonomic failure. Diabetes 57:470-455.

Baldo BA, Daniel RA, Berridge CW, Kelley AE. 2003. Overlapping distributions of orexin/hypocretin- and dopamine-beta-hydroxylase immunoreactive fibers in rat brain regions mediating arousal, motivation, and stress. J Comp Neurol 464:220-237.

Bassett JL, Foote SL. 1992. Distribution of corticotropin-releasing factorlike immunoreactivity in squirrel monkey (Saimiri sciureus) amygdala. J Comp Neurol 323:91-102.

Beck CH, Fibiger HC. 1995. Conditioned fear-induced changes in behavior and in the expression of the immediate early gene c-fos: with and without diazepam pretreatment. J Neurosci 15:709-720.

Beckstead RM. 1984. The thalamostriatal projection in the cat. J Comp Neurol 223:313-346.

Berendse HW, Groenewegen HJ. 1990. Organization of the thalaostriatal projections in the rat, with special emphasis on the ventral striatum. J Comp Neurol 299:187-228.

Berendse HW, Groenewegen HJ. 1991. Restricted cortical termination fields of the midline and intralaminar thalamic nuclei in the rat. Neuroscience 42:73-102.

Bhatnagar S, Dallman M. 1998. Neuroanatomical basis for facilitation of hypothalamic-pituitary-adrenal responses to a novel stressor after chronic stress. Neuroscience 84:1025-1039.

Bhatnagar S, Huber R, Nowak N, Trotter P. 2002. Lesions of the posterior paraventricular thalamus block habituation of hypothalamic-pituitary-adrenal responses to repeated restraint. J Neuroendocrinol 14:403-410.

Bhatnagar S, Huber R, Lazar E, Pych L, Vining C. 2003. Chronic stress alters behavior in the conditioned defensive burying test: role of the posterior paraventricular thalamus. Pharmacol Biochem Behav 76: 343-349.

Boutrel B, Kenny PJ, Specio SE, Martin-Fardon R, Markou A, Koob GF, de Lecea L. 2005. Role for hypocretin in mediating stress-induced reinstatement of cocaine-seeking behavior. Proc Natl Acad Sci U S A 102:19168-19173.

Brady KT, Sinha R. 2005. Co-occurring mental and substance use disorders: the neurobiological effects of chronic stress. Am J Psychiatry 162:1483-1493.

Brauer K, Häusser M, Härtig W, Arendt T. 2000. The core-shell dichotomy of nucleus accumbens in the rhesus monkey as revealed by doubleimmunofluorescence and morphology of cholinergic interneurons. Brain Res 858:151-162.

Breiter HC, Rosen BR. 1999. Functional magnetic resonance imaging of brain reward circuitry in the human. Ann N Y Acad Sci 877:523-547.

Brody AL, Saxena S, Mandelkern MA, Fairbanks LA, Ho ML, Baxter LR. 2001. Brain metabolic changes associated with symptom factor improvement in major depressive disorder. Biol Psychiatry 50:171-178.

Bubser M, Deutch AY. 1999. Stress induces Fos expression in neurons of the thalamic paraventricular nucleus that innervate limbic forebrain sites. Synapse 32:13-22.

Carmichael ST, Price JL. 1994. Architectonic subdivision of the orbital and medial prefrontal cortex in the macaque monkey. J Comp Neurol 346:366-402

Carmichael ST, Price JL. 1995. Limbic connections of the orbital and medial prefrontal cortex in macaque monkeys. J Comp Neurol 363: 615-641.

Cha Cl, Foote SL. 1988. Corticotropin-releasing factor in olivocerebellar climbing-fiber system of monkey (Saimiri sciureus and Macaca fascicularis): parasagittal and regional organization visualized by immunohistochemistry. J Neurosci 8:4121-4137.

Chastrette N, Pfaff DW, Gibbs RB. 1991. Effects of daytime and nighttime stress on Fos-like immunoreactivity in Paraventricular nucleus of the hypothalamus, the habenula, and the posterior paraventricular nucleus of the thalamus. Brain Res 563:339-344.

Chen S, Su HS. 1990. Afferent connections of the thalamic paraventricular and paratenial nuclei in the rat-a retrograde tracing study with iontophoretic application of Fluoro-Gold. Brain Res 522:1-6.

Commons KG, Connolley KR, Valentino RJ. 2003. A neurochemically distinct dorsal raphe-limbic circuit with a potential role in affective disorders. Neuropsychopharmacology 28:206-215. 
Cornwall J, Phillipson OT. 1988. Afferent projections to the dorsal thalamus of the rat as shown by retrograde lectin transport. II. The midline nuclei. Brain Res Bull 21:147-161.

Crocker A, España RA, Papadopoulou M, Saper CB, Faraco J, Sakurai T, Honda M, Mignot E, Scammell TE. 2005. Concomitant loss of dynorphin, NARP, and orexin in narcolepsy. Neurology 65:1184-1188.

Cullinan WE, Herman JP, Battaglia DF, Akil H, Watson SJ. 1995. Pattern and time course of immediate early gene expression in rat brain following acute stress. Neuroscience 64:477-505.

Deadwyler SA, Hayashizaki S, Cheer J, Hampson RE. 2004. Reward, memory and substance abuse: functional neuronal circuits in the nucleus accumbens. Neurosci Biobehav Rev 27:703-711.

Drevets WC. 1999. Prefrontal cortical-amygdalar metabolism in major depression. Ann N Y Acad Sci. 877:614-637.

Drevets WC, Price JL, Simpson JR Jr, Todd RD, Reich T, Vannier M, Raichle ME. 1997. Subgenual prefrontal cortex abnormalities in mood disorders. Nature 386:824-827.

Duncan GE, Johnson KB, Breese GR. 1993. Topographic patterns of brain activity in response to swim stress: assessment by 2-deoxyglucose uptake and expression of Fos-like immunoreactivity. J Neurosci 13 : 3932-3943.

Epstein J, Pan H, Kocsis JH, Yang Y, Butler T, Chusid J, Hochberg H, Murrough J, Strohmayer E, Stern E, Silbersweig DA. 2006. Lack of ventral striatal response to positive stimuli in depressed versus norma subjects. Am J Psychiatry 163:1784-1790.

Ferry AT, Öngür D, An X, Price JL. 2000. Prefrontal cortical projections to the striatum in macaque monkeys: evidence for an organization related to prefrontal networks. J Comp Neurol 425:447-470.

Foote SL, Cha Cl. 1988. Distribution of corticotropin-releasing-factor-like immunoreactivity in brainstem of two monkey species (Saimiri sciureus and Macaca fascicularis): an immunohistochemical study. J Comp Neurol 276:239-264.

Freedman LJ, Cassell MD. 1994. Relationship of thalamic basal forebrain projection neurons to the peptidergic innervation of the midline thalamus. J Comp Neurol 348:321-342.

Gardner KL, Thrivikraman KV, Lightman SL, Plotsky PM, Lowry CA. 2005. Early life experience alters behavior during social defeat: focus on serotonergic systems. Neuroscience 136:181-191.

Giménez-Amaya JM, McFarland NR, de las Heras S, Haber SN. 1995. Organization of thalamic projections to the ventral striatum in the primate. J Comp Neurol 354:127-149.

Grahn RE, Maswood S, McQueen MB, Watkins LR, Maier SF. 1999 Opioid-dependent effects of inescapable shock on escape behavio and conditioned fear responding are mediated by the dorsal raphe nucleus. Behav Brain Res 99:153-167.

Gray TS, Morley JE. 1986. Neuropeptide Y: anatomical distribution and possible function in mammalian nervous system. Life Sci 38:389-401.

Greicius MD, Flores BH, Menon V, Glover GH, Solvason HB, Kenna H, Reiss AL, Schatzberg AF. 2007. Resting-state functional connectivity in major depression: abnormally increased contributions from subgenual cingulate cortex and thalamus. Biol Psychiatry 62:429-437.

Hammack SE, Richey KJ, Schmid MJ, LoPresti ML, Watkins LR, Maier SF. 2002. The role of corticotropin-releasing hormone in the dorsal raphe nucleus in mediating the behavioral consequences of uncontrollable stress. J Neurosci 22:1020-1026.

Harris GC, Wimmer M, Aston-Jones G. 2005. A role for lateral hypothalamic orexin neurons in reward seeking. Nature 437:556-559.

Hasler G, Drevets WC, Manji HK, Charney DS. 2004. Discovering endophenotypes for major depression. Neuropsychopharmacology 29: 1765-1781.

Horvath TL, Diano S, van den Pol AN. 1999. Synaptic interaction between hypocretin (orexin) and neuropeptide $Y$ cells in the rodent and primate hypothalamus: a novel circuit implicated in metabolic and endocrine regulations. J Neurosci 19:1072-1087.

Hsu DT, Price JL. 2007. Midline and intralaminar thalamic connections with the orbital and medial prefrontal networks in macaque monkeys. J Comp Neurol 504:89-111.

Imai H, Steindler DA, Kitai ST. 1986. The organization of divergent axonal projections from the midbrain raphe nuclei in the rat. J Comp Neurol 243:363-380.

Imaki T, Shibasaki T, Hotta M, Demura H. 1993. Intracerebroventricular administration of corticotropin-releasing factor induces c-fos mRNA expression in brain regions related to stress responses: comparison with pattern of c-fos mRNA induction after stress. Brain Res 616:114-125.
Insausti R, Amaral DG, Cowan WM. 1987. The entorhinal cortex of the monkey: III. Subcortical afferents. J Comp Neurol 264:396-408.

Ishibashi M, Takano S, Yanagida H, Takatsuna M, Nakajima K, Oomura Y, Wayner MJ, Sasaki K. 2005. Effects of orexins/hypocretins on neuronal activity in Paraventricular nucleus of the thalamus in rats in vitro. Peptides 26:471-481.

Jaferi A, Nowak N, Bhatnagar S. 2003. Negative feedback functions in chronically stressed rats: role of the posterior paraventricular thalamus. Physiol Behav 78:365-373.

Jones EG. 2007. The thalamus, 2nd ed. New York: Cambridge University Press.

Jones EG, Leavitt RY. 1974. Retrograde axonal transport and the demonstration of non-specific projections to the cerebral cortex and striatum from thalamic intralaminar nuclei in the rat, cat and monkey. J Comp Neurol 154:349-378.

Kelley AE, Baldo BA, Pratt WE. 2005. A proposed hypothalamic-thalamicstriatal axis for the integration of energy balance, arousal, and food reward. J Comp Neurol 493:72-85.

Kirouac GJ, Parsons MP, Li S. 2005. Orexin (hypocretin) innervation of paraventricular nucleus of the thalamus. Brain Res 1059:179-188.

Kritzer MF, Goldman-Rakic PS. 1995. Intrinsic circuit organization of the major layers and sublayers of the dorsolateral prefrontal cortex in the rhesus monkey. J Comp Neurol 359:131-143.

Krout KE, Loewy. 2000a. Parabrachial nucleus projections to midline and intralaminar thalamic nuclei of the rat. J Comp Neurol 428:475-494.

Krout KE, Loewy. 2000b. Periaqueductal gray matter projections to midline and intralaminar thalamic nuclei of the rat. J Comp Neurol 424:111141.

LaMotte CC. 1988. Lamina X of primate spinal cord: distribution of five neuropeptides and serotonin. Neuroscience 25:639-658.

Landry GJ, Yamakawa GR, Mistlberger RE. 2007. Robust food anticipatory circadian rhythms in rats with complete ablation of the thalamic paraventricular nucleus. Brain Res 1141:108-118.

Langevin H, Emson PC. 1982. Distribution of substance P, somatostatin and neurotensin in the human hypothalamus. Brain Res 246:65-69.

Larsen PJ. 1992. Distribution of substance P-immunoreactive elements in the preoptic area and the hypothalamus of the rat. J Comp Neurol 316:287-313.

Lavoie B, Parent A. 1991. Serotoninergic innervation of the thalamus in the primate: an immunohistochemical study. J Comp Neurol 312:1-18.

Li S, Kirouac GJ. 2008. Projections from the paraventricular nucleus of the thalamus to the forebrain, with special emphasis on the extended amygdala. J Comp Neurol 506:263-287.

Maier SF, Watkins LR. 2005. Stressor controllability and learned helplessness: the roles of the dorsal raphe nucleus, serotonin, and corticotropin-releasing factor. Neurosci Biobehav Rev 29:829-841.

Mayberg HS, Liotti M, Brannan SK, McGinnis S, Mahurin RK, Jerabek PA Silva JA, Tekell JL, Martin CC, Lancaster JL, Fox PT. 1999. Reciprocal limbic-cortical function and negative mood: converging PET findings in depression and normal sadness. Am J Psychiatry 156:675-682.

Mayberg HS, Lozano AM, Voon V, McNeely HE, Seminowicz D, Hamani C, Schwalb JM, Kennedy SH. 2005. Deep brain stimulation for treatmentresistant depression. Neuron 45:651-660.

McKenna JT, Vertes RP. 2004. Afferent projections to nucleus reuniens of the thalamus. J Comp Neurol 480:115-142.

Mehler WR. 1980. Subcortical afferent connections of the amygdala in the monkey. J Comp Neurol 190:733-762.

Meloni EG, Reedy CL, Cohen BM, Carlezon WA Jr. 2008. Activation of raphe efferents to the medial prefrontal cortex by corticotropinreleasing factor: correlation with anxiety-like behavior. Biol Psychiatry 63:832-839.

Meredith GE, Pattiselanno A, Groenewegen HJ, Haber SN. 1996. Shell and core in monkey and human nucleus accumbens identified with antibodies to calbindin-D28k. J Comp Neurol 365:628-639.

Moga MM, Weis RP, Moore RY. 1995. Efferent projections of Paraventricular thalamic nucleus in the rat. J Comp Neurol 359:221-238.

Moore RY, Speh JC. 2004. Serotonin innervation of the primate suprachiasmatic nucleus. Brain Res 1010:169-173.

Nakahara K, Fukui K, Murakami N. 2004. Involvement of thalamic paraventricular nucleus in the anticipatory reaction under food restriction in the rat. J Vet Med Sci 66:1297-1300.

Nambu T, Sakurai T, Mizukami K, Hosoya Y, Yanagisawa M, Goto K. 1999. Distribution of orexin neurons in the adult rat brain. Brain Res 827 243-260. 
Nemeroff CB. 1988. The role of corticotropin-releasing factor in Pathogenesis of major depression. Pharmacopsychiatry 21:76-82.

Novak CM, Nunez AA. 1998. Daily rhythms in Fos activity in the rat ventrolateral preoptic area and midline thalamic nuclei. Am J Physiol 275:R1620-1626.

Oldfield BJ, Silverman AJ. 1985. A light microscopic HRP study of limbic projections to the vasopressin-containing nuclear groups of the hypothalamus. Brain Res Bull 14:143-157.

Olszewski J. 1952. The thalamus of the Macaca mulatta. An atlas for use with the stereotaxic instrument. New York: Karger.

Öngür D, Price JL. 2000. The organization of networks within the orbita and medial prefrontal cortex of rats, monkeys and humans. Cereb Cortex 10:206-219.

Öngür D, An X, Price JL. 1998. Prefrontal cortical projections to the hypothalamus in macaque monkeys. J Comp Neurol 401:480-505.

Otake K. 2005. Cholecystokinin and substance P immunoreactive projections to the paraventricular thalamic nucleus in the rat. Neurosci Res 51:383-394.

Otake K, Nakamura Y. 1995. Sites of origin of corticotropin-releasing factor-like immunoreactive projection fibers to Paraventricular thalamic nucleus in the rat. Neurosci Lett 201:84-86.

Otake K, Kin K, Nakamura Y. 2002. Fos expression in afferents to the rat midline thalamus following immobilization stress. Neurosci Res 43: 269-282.

Périco CA, Skaf CR, Yamada A, Duran F, Buchpiguel CA, Castro CC, Soares JC, Busatto GF. 2005. Relationship between regional cerebral blood flow and separate symptom clusters of major depression: a single photon emission computed tomography study using statistical parametric mapping. Neurosci Lett 384:265-270.

Peyron C, Tighe DK, van den Pol AN, de Lecea L, Heller HC, Sutcliffe JG, Kilduff TS. 1998. Neurons containing hypocretin (orexin) project to multiple neuronal systems. J Neurosci 18:9996-10015.

Pierce TL, Wessendorf MW. 2000. Immunocytochemical mapping of endomorphin-2-immunoreactivity in rat brain. J Chem Neuroanat 18: 181-207.

Pizzagalli DA, Oakes TR, Fox AS, Chung MK, Larson CL, Abercrombie HC Schaefer SM, Benca RM, Davidson RJ. 2004. Functional but not structural subgenual prefrontal cortex abnormalities in melancholia. Mol Psychiatry 9:393-405.

Price JL. 2003. Comparative aspects of amygdala connectivity. Ann N Y Acad Sci 985:50-58.

Price JL. 2007. Definition of the orbital cortex in relation to specific connections with limbic and visceral structures and other cortical regions. Ann N Y Acad Sci 1121:54-71.

Price JL, Amaral DG. 1981. An autoradiographic study of the projections of the central nucleus of the monkey amygdala. J Neurosci 1:1242-1259.

Royce GJ, Bromley S, Gracco C, Beckstead RM. 1989. Thalamocortica connections of the rostral intralaminar nuclei: an autoradiographic analysis in the cat. J Comp Neurol 288:555-582.

Sakurai T. 2007. The neural circuit of orexin (hypocretin): maintaining sleep and wakefulness. Nat Rev Neurosci 8:171-181.

Salazar-Juárez A, Escobar C, Aguilar-Roblero R. 2002. Anterior paraventricular thalamus modulates light-induced phase shifts in circadian rhythmicity in rats. Am J Physiol Regul Integr Comp Physiol 283:R897-904.

Sánchez-González MÁ, García-Cabezas MÁ, Rico B, Cavada C. 2005. The primate thalamus is a key target for brain dopamine. J Neurosci 25:6076-6083.

Saper CB, Scammell TE, Lu J. 2005. Hypothalamic regulation of sleep and circadian rhythms. Nature 437:1257-1263.

Saunders RC, Mishkin M, Aggleton JP. 2005. Projections from the entorhinal cortex, perirhinal cortex, presubiculum, and parasubiculum to the medial thalamus in macaque monkeys: identifying different pathways using disconnection techniques. Exp Brain Res 167:1-16.

Schlaepfer TE, Cohen MX, Frick C, Kosel M, Brodesser D, Axmacher N, Joe AY, Kreft M, Lenartz D, Sturm V. 2008. Deep brain stimulation to reward circuitry alleviates anhedonia in refractory major depression. Neuropsychopharmacology 33:368-377.

Semba K, Pastorius J, Wilkinson M, Rusak B. 2001. Sleep deprivationinduced $\mathrm{C}$-fos and junB expression in the rat brain: effects of duration and timing. Behav Brain Res 120:75-86.
Senba E, Matsunaga K, Tohyama M, Noguchi K. 1993. Stress-induced $\mathrm{c}$-fos expression in the rat brain: activation mechanism of sympathetic pathway. Brain Res Bull 31:329-344.

Sharp FR, Sagar SM, Hicks K, Lowenstein D, Hisanaga K. 1991. c-fos mRNA, Fos, and Fos-related antigen induction by hypertonic saline and stress. J Neurosci 11:2321-2331.

Shekhtman E, Geerling JC, Loewy AD. 2007. Aldosterone-sensitive neurons of the nucleus of the solitary tract: multisynaptic pathway to the nucleus accumbens. J Comp Neurol. 501:274-289.

Sica AL, Greenberg HE, Scharf SM, Ruggiero DA. 2000. Chronicintermittent hypoxia induces immediate early gene expression in the midline thalamus and epithalamus. Brain Res 883:224-228.

Siegle GJ, Carter CS, Thase ME. 2006. Use of $\mathrm{fMRI}$ to predict recovery from unipolar depression with cognitive behavior therapy. Am J Psychiatry 163:735-738.

Silverman AJ, Oldfield BJ. 1984. Synaptic input to vasopressin neurons of paraventricular nucleus (PVN). Peptides 5(Suppl 1):139-150.

Spencer SJ, Fox JC, Day TA. 2004. Thalamic paraventricular nucleus lesions facilitate central amygdala neuronal responses to acute psychological stress. Brain Res 997:234-237.

Su HS, Bentivoglio M. 1990. Thalamic midline cell populations projecting to the nucleus accumbens, amygdala, and hippocampus in the rat. J Comp Neurol 297:582-593.

Sutcliffe JG, de Lecea L. 2000. The hypocretins: excitatory neuromodulatory peptides for multiple homeostatic systems, including sleep and feeding. J Neurosci Res 62:161-168.

Swanson LW, Sawchenko PE, Rivier J, Vale WW. 1983. Organization of ovine corticotropin-releasing factor immunoreactive cells and fibers in the rat brain: an immunohistochemical study. Neuroendocrinology 36:165-186.

Szabo J, Cowan WM. 1984. A stereotaxic atlas of the brain of the cynomolgus monkey (Macaca fascicularis). J Comp Neurol 222:265-300.

Teves D, Videen TO, Cryer PE, Powers WJ. 2004. Activation of human medial prefrontal cortex during autonomic responses to hypoglycemia. Proc Natl Acad Sci U S A 101:6217-6221.

Uroz V, Prensa L, Giménez-Amaya JM. 2004. Chemical anatomy of the human paraventricular thalamic nucleus. Synapse 51:173-185.

Van der Werf YD, Witter MP, Groenewegen HJ. 2002. The intralaminar and midline nuclei of the thalamus. Anatomical and functional evidence for participation in processes of arousal and awareness. Brain Res Brain Res Rev 39:107-140.

Van Laere K, Nuttin B, Gabriels L, Dupont P, Rasmussen S, Greenberg BD, Cosyns P. 2006. Metabolic imaging of anterior capsular stimulation in refractory obsessive-compulsive disorder: a key role for the subgenual anterior cingulate and ventral striatum. J Nucl Med 47:740-747.

Vertes RP. 1991. A PHA-L analysis of ascending projections of the dorsal raphe nucleus in the rat. J Comp Neurol 313:643-668.

Vertes RP, Hoover WB. 2008. Projections of the paraventricular and paratenial nuclei of the dorsal midline thalamus in the rat. $\mathrm{J}$ Comp Neurol 508:212-237.

Vertes RP, Fortin WJ, Crane AM. 1999. Projections of the median raphe nucleus in the rat. J Comp Neurol 407:555-582.

Vogt BA, Hof PR, Friedman DP, Sikes RW, Vogt LJ. 2008. Norepinephrinic afferents and cytology of the macaque monkey midline, mediodorsal, and intralaminar thalamic nuclei. Brain Struct Funct 212:465-480.

Watts AG, Swanson LW. 1987. Efferent projections of the suprachiasmatic nucleus: II. Studies using retrograde transport of fluorescent dyes and simultaneous peptide immunohistochemistry in the rat. J Comp Neurol 258:230-252.

Watts AG, Swanson LW, Sanchez-Watts G. 1987. Efferent projections of the suprachiasmatic nucleus: I. Studies using anterograde transport of Phaseolus vulgaris leucoagglutinin in the rat. J Comp Neurol 258:204229.

Westlund KN, Sorkin LS, Ferrington DG, Carlton SM, Willcockson $\mathrm{HH}$, Willis WD. 1990. Serotoninergic and noradrenergic projections to the ventral posterolateral nucleus of the monkey thalamus. J Comp Neurol 295:197-207.

Yanagihara M, Niimi K, Ono K. 1987. Thalamic projections to the hippocampal and entorhinal areas in the cat. J Comp Neurol 266:122-141.

Zhang DX, Bertram EH. 2002. Midline thalamic region: widespread excitatory input to the entorhinal cortex and amygdala. J Neurosci 15: 3277-3284. 